\title{
On the Existence and Properties of the Rotationally Symmetric \\ Equilibrium States of Compressible Barotropic Self-Gravitating Fluids
}

\section{Gerhard Ströhmer AND WojcIech ZajacZKowski}

\begin{abstract}
The present paper proves that the spherically symmetric solution of the Lane-Emden equation is a strict local minimum of the energy even with respect to perturbations which are not spherically symmetric. This energy includes a term representing a fixed outside pressure.
\end{abstract}

\section{INTRODUCTION}

Up to a constant factor the free energy of a polytropic self-gravitating gas with the equation of state $p(\rho)=\rho^{\varkappa}$ contained in a domain $\Omega \subset \mathbb{R}^{3}$ with a pressure $p_{0}$ outside $\Omega$ is given by

$$
E(\rho, \Omega)=\int_{\Omega}\left((\varkappa-1) p_{0}+\rho^{\varkappa}(x)\right) d x-\frac{A}{4 \pi} \int_{\Omega} \int_{\Omega} \frac{\rho(x) \rho(y)}{|x-y|} d x d y .
$$

Here $\rho(x) \geq 0$ is the mass density and so

$$
M=\int_{\Omega} \rho(x) d x
$$

is the mass of the gas in $\Omega$. Under the assumption that $\varkappa>\frac{4}{3}$, we will show that given the total mass there is a ball of a uniquely determined radius $R$ and a uniquely determined radially symmetric density distribution in this ball minimizing energy among all radially symmetric distributions in balls. It also is 
a strict relative minimum of the energy with respect to all nearby distributions and domains with the same total mass, regardless of the symmetry.

This result will be used in later papers (see [25], [26]) to prove that the equations of compressible fluid flow with suitable boundary conditions on the free boundary have solutions existing for all time for initial values which are small perturbations of this state, and that this state is asymptotically stable. We are using a positive outside pressure because the equations of fluid dynamics are not a good model for very small densities. Instead of making the whole system unduly complicated by using the Boltzmann equation for regions of very low density, we just chose to simulate the rarefied gas in these regions by a massless entity exerting a fixed pressure. In a number of papers about solutions of the equations of compressible fluid flow with or without the influence of gravity either this or surface tension was used also to keep the density from becoming zero (see, e.g. [32], [33]).

If, in a sense we will soon make precise, $\rho_{0}$ is a density distribution on a ball $B_{R_{0}}$, and both together have minimal energy, then (see Lemma 4) they fulfill the condition

$$
\varkappa \rho_{0}^{\varkappa-1}(x)=\frac{A}{2 \pi} \int_{B_{R}} \frac{\rho_{0}(y)}{|x-y|} d y+C \text { on } \bar{B}_{R}, \quad \text { and } \quad p_{0}=\rho_{0}^{\varkappa}(x) \text { on } \partial B_{R_{0}}
$$

with a suitable constant $C$. Taking the Laplace operator of both sides of the first equation, we get

$$
\Delta\left(\rho_{0}^{\varkappa-1}(x)\right)=-\frac{2 A}{\varkappa} \rho_{0}(x),
$$

or, substituting $s_{0}(x)=\rho_{0}^{\varkappa-1}(x)$,

$$
\Delta\left(s_{0}(x)\right)=-\frac{2 A}{\varkappa} s_{0}^{1 /(\varkappa-1)}(x) .
$$

Under the assumption of radial symmetry $\rho_{0}(x)=\rho_{*}(|x|)$ we have

$$
\frac{d^{2}}{(d r)^{2}}\left(\rho_{*}^{\varkappa-1}\right)(r)+\frac{2}{r} \frac{d}{d r}\left(\rho_{*}^{\varkappa-1}\right)(r)=-\frac{2 A}{\varkappa} \rho_{*}(r),
$$

and with $s(r)=\rho_{*}^{\varkappa-1}(r)$ we get

$$
s^{\prime \prime}(r)+\frac{2}{r} s^{\prime}(r)=-\frac{2 A}{\varkappa}(s(r))^{1 /(\varkappa-1)} .
$$

This is known as the Lane-Emden equation, and was used in 1870 by Lane [12] to estimate the pressure and temperature at the center of the sun. It indicates the strong interest of astrophysicists in such problems which persists to this day. The book by R. Emden [7] (1907) summarized the research in this subject up to the time and his own contributions, and it gives a proof for the existence of a solution of such an equation representing a given mass. It should be noted 
that except for a few special $\varkappa$ there does not seem to be a general explicit solution (see [15]), and so this equation has been the subject of many numerical calculations.

While the early research focused on the existence of spherically symmetric solutions, later the locally minimizing character was studied by astrophysicists, in particular Lynden-Bell and Sanitt [14]. Although the arguments in this paper are mathematically quite sophisticated, the authors leave many questions open and stop short of a rigorous argument, and in any case do not consistently consider the problem with a given outside pressure we are dealing with here.

Existence and properties of solutions of equations similar to (2) have been studied fairly frequently (see [18],[11], and the references contained therein), and are, e.g., covered by the result of Gidas, Ni, Nirenberg, [9], but the questions addressed in these papers do not seem to have an immediate bearing on our problem.

To our knowledge the result about the locally minimizing character of the radially symmetric solution for this problem with outside pressure is new, while many of the other considerations are included to make this paper reasonably self-contained.

Now let us move to a higher degree of precision. The statement that the domain $\Omega$ is near $B_{R}$ will at least include the existence of an orientation-preserving diffeomorphism $T: \bar{B}_{R} \rightarrow \bar{\Omega}$. Then we can replace the density $\rho$ on $\bar{\Omega}$ by a density $\hat{\rho}$ on $\bar{B}_{R}$ defined by the formula

$$
\widehat{\rho}(x)=J_{T}(x) \rho(T(x)),
$$

and so

$$
\int_{B_{R}} \widehat{\rho}(x) d x=\int_{B_{R}} J_{T}(x) \rho(T(x)) d x=\int_{\Omega} \rho(x) d x=M
$$

by the transformation formula. By the same theorem we can also obtain a modified form of the energy in terms of $\widehat{\rho}$ and $T$ as follows:

$$
\begin{aligned}
E(\rho, \Omega)= & \int_{\Omega}\left((\varkappa-1) p_{0}+\rho^{\varkappa}(x)\right) d x-\frac{A}{4 \pi} \int_{\Omega} \int_{\Omega} \frac{\rho(x) \rho(y)}{|x-y|} d x d y \\
=p_{0}(\varkappa-1) \int_{B_{R}} J_{T} d x+ & \int_{B_{R}} \hat{\rho}^{\varkappa}(x) J_{T}^{-\varkappa+1} d x \\
& \quad-\frac{A}{4 \pi} \int_{B_{R}} \int_{B_{R}} \frac{\widehat{\rho}(x) \widehat{\rho}(y)}{|T(x)-T(y)|} d x d y .
\end{aligned}
$$

Now we can define $I(\widehat{\rho}, T)=E(\rho, \Omega)$. We can consider $I$ as a non-linear functional defined on the set

$$
\begin{aligned}
D(I)=\{\widehat{\rho} & \left.\in C^{0}\left(\bar{B}_{R}\right): \widehat{\rho}(x)>0 \text { for all } x \in \bar{B}_{R}\right\} \\
& \times\left\{T \in C^{1}\left(\bar{B}_{R}\right): T \text { is one-to one and } J_{T}(x)>0 \text { for } x \in \bar{B}_{R}\right\} .
\end{aligned}
$$


We will show that this functional is at least three times continuously differentiable.

In this paper we will prove, as already mentioned, that for any given positive $p_{0}$ and $M$ there is a spherically symmetric solution $\rho_{0}$ of (3) so that $\rho_{0}$ and the identity transformation form a stationary point of the integral $I(\widehat{\rho}, T)$. We go beyond this by showing that this solution is indeed a local minimum of the energy among all nearby domains and density distributions, even if these are not spherically symmetric. Now in contrast to the case of the equilibria of incompressible fluids we must consider variations of the domain and the density. If we do not restrict our possibilities, we will find many variations of the domain whose effect is canceled out by variations of the density. Therefore we will restrict ourselves to the following class of variations of the domain for much of this paper. Let $f: S^{2} \rightarrow \mathbb{R}$ be continuously differentiable, and let

$$
T(x)=\left[1+f\left(\frac{x}{|x|}\right) \frac{|x|^{4}}{R^{4}}\right] x=[1+g(x)] x .
$$

The factor $|x|^{4}$ is introduced here solely to make $T$ sufficiently differentiable at $x=0$. It is easy to see that every domain close to $B_{R}$ in the sense that $\Omega=\widetilde{T}\left(B_{R}\right)$ with a general transformation close to the identity in the $C^{1}$-norm can be written as $T\left(B_{R}\right)$ with a $T$ of the type above. By a translation we can also make sure that $f$ is orthogonal in $L_{2}\left(S^{2}\right)$ to all spherical harmonics of order one, as we shall show in Lemma 25.

The result is as follows.

Theorem 1. Assume $M>0$ and $p_{0}>0$ are given. Then there is exactly one number $R_{0}>0$ and one function $\rho_{*}:\left[0, R_{0}\right] \rightarrow[0,+\infty)$ with $\rho_{*} \in$ $C^{\infty}\left(\left[0, R_{0}\right]\right)$ such that with $\rho_{0}(x)=\rho_{*}(|x|)$ we have

$$
\int_{B_{R_{0}}} \rho_{0}(x) d x=M
$$

$\rho_{*}^{\varkappa}\left(R_{0}\right)=p_{0}$, and

$$
\frac{d^{2}}{(d r)^{2}}\left(\rho_{*}^{\varkappa-1}(r)\right)+\frac{2}{r} \frac{d}{d r}\left(\rho_{*}^{\varkappa-1}(r)\right)=-2 \frac{A}{\varkappa} \rho_{*}(r) .
$$

This solution also has the property that there is an $\varepsilon>0$ and an $\eta>0$ such that if

$$
\begin{gathered}
\left\|T-\operatorname{id}_{B_{R_{0}}}\right\|_{C^{1}\left(B_{R_{0}}\right)}+\left\|T-\operatorname{id}_{B_{R_{0}}}\right\|_{H_{2}^{2}\left(B_{R_{0}}\right)} \leq \varepsilon, \\
\int_{B_{R}} \hat{\rho} d x=M \quad \text { and } \quad\left\|\widehat{\rho}-\rho_{0}\right\|_{C^{0}\left(B_{R_{0}}\right)} \leq \varepsilon,
\end{gathered}
$$


then

$$
I(\widehat{\rho}, T) \geq I\left(\rho_{0}, \mathrm{id}\right)+\eta\left[\left\|\widehat{\rho}-\rho_{0}\right\|_{L_{2}\left(B_{R}\right)}^{2}+\|f\|_{L_{2}\left(S^{2}\right)}^{2}\right]
$$

for all transformations of the above type if $f$ is perpendicular to all spherical harmonics of order one.

\section{Notation}

We use $C$ to denote a generic constant. For any open subset $\Omega$ of $\mathbb{R}^{3}$ we denote by $L_{p}(\Omega)$ the Lebesgue-measurable functions whose $p-t h$ power is integrable over $\Omega$. For any compact surface in $\mathbb{R}^{3}$ we use integrability with respect to the surface area. Again for any open subset $\Omega$ of $\mathbb{R}^{3}$ we denote by $C^{k}(\Omega)(k=0,1, \ldots)$ the functions which are $k$-times continuously differentiable in $\Omega$, while $C^{k}(\bar{\Omega})$ denotes those elements of $C^{k}(\Omega)$ whose derivatives up to order $k$ are continuous up to the boundary. By $H_{p}^{k}(\Omega)$ we denote those functions in $L_{p}(\Omega)$ which have weak derivatives up to order $k$ which are also in $L_{p}(\Omega)$. The set $\Omega$ will often be omitted if there is no danger of confusion. The notation for Sobolev spaces of fractional order follows that of [27]. For any local transformation $T$ from a domain in $\mathbb{R}^{n}$ to another $J_{T}$ denotes the Jacobian determinant.

\section{Some Preliminary Considerations}

This sections deals with the differentiability properties of $I$ and examines the first derivative a little more closely. Through much of this paper, as already mentioned, we will consider transformations $T$ of the form $T(x)=(1+g(x)) x$. For this reason we want to calculate the functional determinant of such $T$.

Lemma 1. If $g \in C^{1}\left(\bar{B}_{R}\right)$ is a scalar function and $T(x)=(1+g(x)) x$, then

$$
J_{T}(x)=(1+g)^{2}\left(1+g+r \frac{\partial g}{\partial r}\right) .
$$

Proof. Let $O$ be any orthogonal $3 \times 3$ matrix and $T^{\prime}(x)=O^{T} T(O x)$. Then $J_{T^{\prime}}(x)=J_{T}(O x)$ and $T^{\prime}(x)=(1+g(O x)) x$. Now let $x=(0,0, r)$ and $h(x)=$ $1+g(O x)$. Then

$$
\frac{\partial T_{i}^{\prime}}{\partial x_{j}}(x)=\frac{\partial h(x)}{\partial x_{j}} x_{i}+\delta_{i j} h(x)
$$

and so

$$
\left[\frac{\partial T_{i}^{\prime}}{\partial x_{j}}\right]_{i j}(x)=\left[\begin{array}{ccc}
h(x) & 0 & 0 \\
0 & h(x) & 0 \\
\frac{\partial h(x)}{\partial x_{1}} x_{3} & \frac{\partial h(x)}{\partial x_{2}} x_{3} & \frac{\partial h(x)}{\partial x_{3}} x_{3}+h
\end{array}\right]
$$


Therefore $J_{T}(O x)=J_{T^{\prime}}(x)=h^{2}\left(h+r \frac{\partial h}{\partial r}\right)(x)=(1+g)^{2}\left(1+g+r \frac{\partial g}{\partial r}\right)(O x)$ for $x=(0,0, r)$ and arbitrary $O$. This implies our claim.

In what follows we will think of second derivatives as quadratic forms rather than bilinear forms. For an introduction to calculus of mappings between Banach spaces see [13].

Lemma 2. The functional $I: D(I) \rightarrow \mathbb{R}$ belongs to $C^{3}(D(I))$.

Let $\rho_{0}$ be a radially symmetric solution of (1). If we confine ourselves to the transformations of the form

$$
T(x)=\left[1+f\left(\frac{x}{|x|}\right)\left(\frac{|x|}{R}\right)^{4}\right] x=[1+g(x)] x,
$$

then there is an $\varepsilon>0$ and a constant $C$ such that if

$$
\|\varphi\|_{C^{0}}+\|T-\mathrm{id}\|_{C^{1}}+\|T-\mathrm{id}\|_{H^{2}} \leq \varepsilon,
$$

then with $u=T-\mathrm{id}=g(x) x$, and $\varphi=\rho-\rho_{0}$ we have for $\left|t_{0}\right| \leq 1$

$$
\begin{aligned}
& \left|\left(\frac{d}{d t}\right)^{3} I\left(\rho_{0}+t \varphi, \mathrm{id}+t u\right)\right|_{t=t_{0}} \mid \\
& \quad \leq C\left(\|\varphi\|_{C^{0}}+\|u\|_{H^{2}}+\|u\|_{C^{1}}\right)\left(\|u\|_{L_{2}}^{2}+\|\varphi\|_{L_{2}}^{2}\right) .
\end{aligned}
$$

Proof. For $(\widehat{\rho}, T) \in D(I)$ we have $I(\widehat{\rho}, T)=I_{1}(\widehat{\rho}, T)+I_{2}(\widehat{\rho}, T)$ with

$$
I_{1}(\widehat{\rho}, T)=p_{0}(\varkappa-1) \int_{B_{R}} J_{T} d x+\int_{B_{R}} \widehat{\rho}^{\varkappa}(x) J_{T}^{-\varkappa+1} d x
$$

and

$$
I_{2}(\widehat{\rho}, T)=-\frac{A}{4 \pi} \int_{B_{R}} \int_{B_{R}} \frac{\widehat{\rho}(x) \widehat{\rho}(y)}{|T(x)-T(y)|} d x d y .
$$

Now the differentiability of the first expression is rather obvious as integration is a bounded linear functional on $C^{0}\left(\bar{B}_{R}\right)$ and the expressions that form the integrands are even analytic functions of $\hat{\rho}$ and $\nabla T$.

To show the differentiability properties of the second expression consider the approximating functionals

$$
I_{2}^{\delta}(\widehat{\rho}, T)=-\frac{A}{4 \pi} \iint_{D_{\delta}} \frac{1}{|T(x)-T(y)|} \widehat{\rho}(x) \widehat{\rho}(y) d x d y
$$


for $\delta>0$ with $D_{\delta}=\left\{(x, y): x, y \in B_{R}\right.$ and $\left.|x-y|>\delta\right\}$. Now let $T_{0} \in C^{1}\left(\bar{B}_{R}\right)$ be a diffeomorphism. As the diffeomorphisms form an open set in $C^{1}\left(\bar{B}_{R}\right)$ there is an $\varepsilon>0$ such that for all $T$ with $\left\|T-T_{0}\right\|_{C^{1}}<\varepsilon$ we have

$$
C_{2}|x-y| \geq|T(x)-T(y)|\left|\geq C_{1}\right| x-y \mid \quad\left(x, y \in \bar{B}_{R}\right)
$$

with $C_{2}>C_{1}>0$. This means that $I_{2}^{\delta}$ will be infinitely differentiable in a small $C^{1}$-neighborhood of $T_{0}$ for $\delta>0$, and we will see that the first three derivatives converge uniformly to those of $I_{2}(\widehat{\rho}, T)$ as $\delta \rightarrow \infty$.

Now for any such $T_{1}$ with $\left\|T_{1}-T_{0}\right\|_{C^{1}}<\varepsilon$ let

$$
\begin{gathered}
T_{t}(x)=t T_{1}(x)+(1-t) T_{0}(x), \\
S(x, y, t)=t\left(T_{1}(x)-T_{1}(y)\right)+(1-t)\left(T_{0}(x)-T_{0}(y)\right)=T_{t}(x)-T_{t}(y), \\
\rho(t, x)=(1-t) \hat{\rho}(x)+t \rho_{1}(x),
\end{gathered}
$$

and

$$
\begin{aligned}
& u(x)=T_{1}(x)-T_{0}(x), \\
& \varphi(x)=\rho_{1}(x)-\widehat{\rho}(x) .
\end{aligned}
$$

As the ball around $T_{0}$ of radius $\varepsilon$ in $C^{1}$ is convex, $T_{t}$ also belongs to it, and so we have that

$$
C_{2}|x-y| \geq|S(x, y, t)| \geq C_{1}|x-y| \quad\left(x, y \in \bar{B}_{R}\right) .
$$

Also,

$$
\begin{aligned}
\frac{\partial}{\partial t}\left(\frac{1}{|S(x, y, t)|}\right) & =-\frac{1}{|S|^{3}} S \cdot S_{t} \\
\frac{\partial^{2}}{(\partial t)^{2}}\left(\frac{1}{|S(x, y, t)|}\right) & =\frac{3}{|S|^{5}}\left(S \cdot S_{t}\right)^{2}-\frac{1}{|S|^{3}} S_{t}^{2}, \text { and } \\
\frac{\partial^{3}}{(\partial t)^{3}}\left(\frac{1}{|S(x, y, t)|}\right) & =-\frac{15}{|S|^{7}}\left(S \cdot S_{t}\right)^{3}+\frac{9}{|S|^{5}}\left(S \cdot S_{t}\right) S_{t}^{2} .
\end{aligned}
$$

As also $\left|S_{t}\right| \leq C|x-y|$, we get that

$$
\begin{aligned}
& \left|\frac{\partial^{k}}{(\partial t)^{k}}\left(\frac{1}{|S(x, y, t)|}\right)\right| \leq \frac{C}{|x-y|} \quad \text { for } k=1,2,3, \\
& \left|\frac{\partial^{k}}{(\partial t)^{k}}\left(\frac{1}{|S(x, y, t)|}\right)\right| \leq \frac{C}{|x-y|^{\ell+1}}\left|S_{t}\right|^{\ell} \quad \text { for } k=1,2,3, \quad \ell=0,1, \ldots, k .
\end{aligned}
$$


Also note that $S_{t}(x, y, t)=u(x)-u(y)$. From the first inequality it is very easy to derive the differentiability properties in $C^{1}$ of the second expression by proving convergence of the approximating functions. To get estimate (5), we first notice from Lemma 1 that then as $T(x)=(1+g(x)) x$ we have

$$
\begin{aligned}
I_{1}\left(\rho_{0}+t \varphi, \mathrm{id}+t u\right)=p_{0}(\varkappa- & 1) \int_{B_{R}}(1+t g)^{2}(1+5 t g) d x \\
& +\int_{B_{R}}\left(\rho_{0}+t \varphi\right)^{\varkappa}\left((1+t g)^{2}(1+5 t g)\right)^{-\varkappa+1} d x,
\end{aligned}
$$

which obviously has this property. For $I_{2}$ note that here $T_{t}(x)=(1+t g(x)) x$ and

$$
\begin{aligned}
\frac{d^{3}}{(d t)^{3}}\left(-I_{2}\left(\rho, T_{t}\right)\right)= & 3 \int_{B_{R}} \int_{B_{R}} \frac{\partial^{2}}{(\partial t)^{2}}(\rho(x, t) \rho(y, t)) \frac{\partial}{\partial t} \frac{1}{\left|T_{t}(x)-T_{t}(y)\right|} d x d y \\
& +3 \int_{B_{R}} \int_{B_{R}} \frac{\partial}{\partial t}(\rho(x, t) \rho(y, t)) \frac{\partial^{2}}{(\partial t)^{2}} \frac{1}{\left|T_{t}(x)-T_{t}(y)\right|} d x d y \\
& +\int_{B_{R}} \int_{B_{R}} \rho(x, t) \rho(y, t) \frac{\partial^{3}}{(\partial t)^{3}} \frac{1}{\left|T_{t}(x)-T_{t}(y)\right|} d x d y \\
= & J_{1}+J_{2}+J_{3} .
\end{aligned}
$$

Now we have

$$
\begin{aligned}
\left|J_{1}\right| & \leq C \int_{B_{R}} \int_{B_{R}}|\varphi(x) \varphi(y)| \frac{\left|S_{t}\right|}{|x-y|^{2}} d x d y \\
& \leq C \int_{B_{R}} \int_{B_{R}}|\varphi(x) \varphi(y)| \frac{|u(x)-u(y)|}{|x-y|^{2}} d x d y \\
& \leq C\|u\|_{C^{1}} \int_{B_{R}} \int_{B_{R}} \frac{|\varphi(x) \varphi(y)|}{|x-y|} d x d y \\
& \leq C\|u\|_{C^{1}}\|u\|_{L_{2}}\|\varphi\|_{L_{2}},
\end{aligned}
$$

which takes care of the first expression. We also have

$$
\begin{aligned}
\left|J_{2}\right| & \leq C \int_{B_{R}} \int_{B_{R}}|\varphi(y)| \frac{|u(x)-u(y)|^{2}}{|x-y|^{3}} d x d y \\
& \leq C\|u\|_{C^{1}} \int_{B_{R}} \int_{B_{R}}|\varphi(y)| \frac{|u(x)-u(y)|}{|x-y|^{2}} d x d y \leq
\end{aligned}
$$




$$
\begin{aligned}
& \leq C\|u\|_{C^{1}} \int_{B_{R}} \int_{B_{R}} \frac{|u(x)|+|u(y)|}{|x-y|^{2}}|\varphi(y)| d x d y \\
& \leq C\|u\|_{C^{1}}\left[\int_{B_{R}} \int_{B_{R}} \frac{|u(x)|}{|x-y|^{2}}|\varphi(y)| d x d y\right. \\
& \left.\quad+\int_{B_{R}} \int_{B_{R}} \frac{|u(y)|}{|x-y|^{2}}|\varphi(y)| d x d y\right] \\
& \leq C\|u\|_{C^{1}} \quad\|u\|_{L_{2}} \quad\|\varphi\|_{L_{2}} .
\end{aligned}
$$

To estimate the last expression we need to remember $u=f(x /|x|)(|x| / R)^{4} x$, so that we can use embedding theorems for two-dimensional space for inequality (6). We have

$$
\begin{aligned}
\left|J_{3}\right| & \leq C \int_{B_{R}} \int_{B_{R}} \frac{|u(x)-u(y)|^{3}}{|x-y|^{4}} d x d y \leq C\|u\|_{W_{3}^{1 / 3}\left(B_{R}\right)}^{3} \\
& \leq C\|u\|_{W_{3}^{1 / 3}\left(\partial B_{R}\right)}^{3} \leq C\|u\|_{W_{2}^{2 / 3}\left(\partial B_{R}\right)}^{3} \\
& \leq C\|u\|_{W_{2}^{2 / 3}\left(B_{R}\right)}^{3} \leq C\|u\|_{L_{2}\left(B_{R}\right)}^{2}\|u\|_{W_{2}^{2}\left(B_{R}\right)}
\end{aligned}
$$

using Remark 2 (Section 4.41, p. 323), Theorem 1 in Section 4.3.1, and Remark 1 (Section 4.6.1, p. 328), all in [27]. Then we have proved our claim.

Now we define the first and second variations, or Gâteau derivatives, denoted in a form more in tune with what is usual in the calculus of variations.

Definition 1. Let $u \in C^{1}\left(\bar{B}_{R}\right)$ and $\varphi, \rho \in C^{0}\left(\bar{B}_{R}\right)$. Let also $\rho(x)>0$ for all $x \in \bar{B}_{R}$. Then let

$$
V_{k}(\rho, u, \varphi)=\left(\frac{d^{k}}{(d t)^{k}} I(\rho+t \varphi, \mathrm{id}+t u)\right)(0) \text { for } k=1,2 .
$$

Note that

$$
V_{k}(\rho, u, \varphi)=\left(D^{k} I\right)(\rho)(u, \varphi) .
$$

Now we want to change the form of the first variation to make it easier to obtain certain conclusions. To this end we assume $\rho_{0} \in C^{0}\left(\bar{B}_{R}\right)$ and $\rho_{0}(x)>0$ on $\bar{B}_{R}$. we have

Lemma 3. If $u \in C^{1}\left(\bar{B}_{R}\right)$ and $\varphi \in C^{0}\left(\bar{B}_{R}\right)$, then with $T_{t}(x)=x+t u(x)$

$$
\left.\frac{d}{d t}\right|_{t=0} J_{T_{t}}=\operatorname{div}(u)
$$

and 


$$
\begin{aligned}
V_{1}(\rho, \varphi, u)=(\varkappa-1) & \int_{\partial B_{R}}\left(p_{0}-\rho_{0}^{\varkappa}\right)(u \cdot n) d \sigma \\
+ & \int_{B_{R}}\left[\varphi(x)+\rho_{0}(x)\left(u(x) \cdot \nabla_{x}\right)\right] \\
& \times\left[\varkappa \rho_{0}^{\varkappa-1}(x)-\frac{A}{2 \pi} \int_{B_{R}} \frac{\rho_{0}(y)}{|x-y|} d y\right] d x .
\end{aligned}
$$

Proof. The first claim can be verified by straightforward calculation. Further, as we have

$$
\begin{aligned}
I\left(\rho(., t), T_{t}\right)=p_{0}(\varkappa-1) & \int_{B_{R}} J_{T_{t}} d x \\
& +\int_{B_{R}} \rho^{\varkappa}(x, t) J_{T_{t}}^{-\varkappa+1} d x \\
& -\frac{A}{4 \pi} \int_{B_{R}} \int_{B_{R}} \frac{\rho(x, t) \rho(y, t)}{\left|T_{t}(x)-T_{t}(y)\right|} d x d y,
\end{aligned}
$$

we obtain

$$
\begin{aligned}
V_{1}(\rho, \varphi, u)=\varkappa \int_{B_{R}} \rho_{0}^{\varkappa-1} \varphi d x-\frac{2 A}{4 \pi} \int_{B_{R}} \int_{B_{R}} \frac{\rho_{0}(x) \varphi(y)}{|x-y|} d x d y \\
+(\varkappa-1) p_{0} \int_{B_{R}} \operatorname{div}(u) d x+(1-\varkappa) \int_{B_{R}} \rho_{0}^{\varkappa}(x) \operatorname{div}(u) d x \\
+\frac{A}{4 \pi} \int_{B_{R}} \int_{B_{R}} \frac{\rho_{0}(x) \rho_{0}(y)}{|x-y|^{3}}(x-y) \cdot(u(x)-u(y)) d x d y .
\end{aligned}
$$

Now

$$
\begin{array}{r}
\frac{1}{2} \int_{B_{R}} \int_{B_{R}} \frac{\rho_{0}(x) \rho_{0}(y)}{|x-y|^{3}}(x-y) \cdot(u(x)-u(y)) d x d y \\
\quad=\int_{B_{R}} \int_{B_{R}} \frac{\rho_{0}(x) \rho_{0}(y)}{|x-y|^{3}}(x-y) \cdot u(x) d x d y \\
=-\int_{B_{R}} \rho_{0}(x)\left(u \cdot \nabla_{x}\right) \int_{B_{R}} \frac{\rho_{0}(y)}{|x-y|} d y d x
\end{array}
$$


Also

$$
\begin{aligned}
p_{0} \int_{B_{R}} \operatorname{div}(u) d x-\int_{B_{R}} \rho_{0}^{\varkappa} \operatorname{div}(u) d x \\
\quad=p_{0} \int_{B_{R}} \operatorname{div}(u) d x-\int_{B_{R}} \operatorname{div}\left(\rho_{0}^{\varkappa} u\right)-(u \cdot \nabla) \rho_{0}^{\varkappa} d x \\
\quad=\int_{\partial B_{R}}\left(p_{0}-\rho_{0}^{\varkappa}\right)(u \cdot n) d \sigma+\frac{\varkappa}{\varkappa-1} \int_{B_{R}} \rho_{0}\left(u(x) \cdot \nabla_{x}\right) \rho_{0}^{\varkappa-1}(x) d x .
\end{aligned}
$$

Putting it all together, we get our claim. Using this result, we get the following:

Lemma 4. Under the assumptions of Lemma 3 we have that $V_{1}\left(\rho_{0}, u, \varphi\right)=$ 0 for all $u$ and all $\varphi$ such that $\int_{B_{R}} \varphi d x=0$, is equivalent to $\rho_{0}^{\varkappa-1} \in C^{2}\left(\bar{B}_{R}\right)$ and

$$
\varkappa \rho_{0}^{\varkappa-1}(x)=\frac{A}{2 \pi} \int_{B_{R}} \frac{\rho_{0}(y)}{|x-y|} d y+C \text { on } \bar{B}_{R}, \quad \text { and } \quad p_{0}=\rho_{0}^{\varkappa}(x) \text { on } \partial B_{R} .
$$

Proof. First we are considering $u=0$ only. As we have the constraint $\int_{B_{R}} \varphi d x=0$, the vanishing of the variation leads to

$$
\varkappa \rho_{0}^{\varkappa-1}(x)=\frac{A}{2 \pi} \int_{B_{R}} \frac{\rho_{0}(y)}{|x-y|} d y+C,
$$

and immediately $\rho_{0}^{\varkappa-1} \in C^{1}\left(\bar{B}_{R}\right) \cap C^{2}\left(B_{R}\right)$. Then

$$
V_{1}(\varphi, u)=(\varkappa-1) \int_{\partial B_{R}}\left(p_{0}-\rho_{0}^{\varkappa}\right)(u \cdot n) d \sigma
$$

and so of course $p_{0}=\rho_{0}^{\varkappa}$ on $\partial B_{R}$, which implies $\rho_{0}^{\varkappa-1} \in C^{2}\left(\bar{B}_{R}\right)$ by [10], Theorem 4.12.

Let us finally state a result about spherical harmonics which we will use several times.

Lemma 5. Let $Y: S^{2} \rightarrow \mathbb{R}$ be a spherical harmonic of degree $n$. Then with $S_{r}=\left\{x \in \mathbb{R}^{3}:|x|=r\right\}$ we have

$$
\int_{S_{r}} \frac{Y\left(\frac{y}{|y|}\right)}{|x-y|} d \sigma= \begin{cases}\frac{4 \pi r}{2 n+1} Y\left(\frac{x}{|x|}\right)\left(\frac{|x|}{r}\right)^{-n-1} & \text { for }|x| \geq r \\ \frac{4 \pi r}{2 n+1} Y\left(\frac{x}{|x|}\right)\left(\frac{|x|}{r}\right)^{n} & \text { for }|x| \leq r\end{cases}
$$


Proof. With some notational differences this is the content of equations (97) and (98) on p. 522 ff. of Section 138 in Chapter VI, Section 1 of Volume III of Smirnov ([23]).

\section{The SECOND VARIATION}

We will bring the second variation into a more suitable form assuming

$$
\varkappa \rho_{0}^{\varkappa-1}(x)=\frac{A}{2 \pi} \int_{B_{R}} \frac{\rho_{0}(y)}{|x-y|} d y+C
$$

in $\Omega$ and $\left(p_{0}-\rho_{0}^{\varkappa}(x)\right) u(x)=0$ on $\partial B_{R}$. We do this for the most general kind of transformation in part because it makes some of our later considerations easier, in part because it is instructive and not any more difficult than the special case. This means we are considering $T_{t}(x)=x+t u(x)$ and $\rho(x, t)=\rho_{0}(x)+t \varphi(x)$. Throughout Lemma 6 to Lemma 12 we assume $u \in C^{2}\left(\bar{B}_{R}\right)$ and $\varphi \in C^{0}\left(\bar{B}_{R}\right)$.

For the second variations we first note the second derivative of $J_{T_{t}}$ at $t=0$.

Lemma 6. We have at $t=0$ that

$$
\frac{d^{2}}{(d t)^{2}} J_{T_{t}}=\operatorname{div}(\operatorname{div}(u) u-(u \cdot \nabla u)) .
$$

Proof. By a straightforward calculation one obtains

$$
\begin{aligned}
\frac{d^{2}}{(d t)^{2}} J_{T_{t}} & =2\left(u_{x_{2}}^{2} u_{x_{3}}^{3}-u_{x_{3}}^{2} u_{x_{2}}^{3}+u_{x_{1}}^{1} u_{x_{3}}^{3}-u_{x_{1}}^{3} u_{x_{3}}^{1}+u_{x_{1}}^{1} u_{x_{2}}^{2}-u_{x_{2}}^{1} u_{x_{1}}^{2}\right) \\
& =2 \sum_{i<j}\left(u_{x_{i}}^{i} u_{x_{j}}^{j}-u_{x_{j}}^{i} u_{x_{i}}^{j}\right)=\sum_{i \neq j}\left(u_{x_{i}}^{i} u_{x_{j}}^{j}-u_{x_{j}}^{i} u_{x_{i}}^{j}\right) \\
& =\sum_{i, j}\left(u_{x_{i}}^{i} u_{x_{j}}^{j}-u_{x_{j}}^{i} u_{x_{i}}^{j}\right) \\
& =\sum_{i, j}\left[u_{x_{i}}^{i} u_{x_{j}}^{j}+u_{x_{i} x_{j}}^{i} u^{j}-u^{i} u_{x_{i} x_{j}}^{j}-u_{x_{j}}^{i} u_{x_{i}}^{j}\right] \\
& =\sum_{i, j}\left[\left(u_{x_{i}}^{i} u^{j}\right)_{x_{j}}-\left(u^{i} u_{x_{i}}^{j}\right)_{x_{j}}\right]=\operatorname{div}(\operatorname{div}(u) u-(u \cdot \nabla u))
\end{aligned}
$$

To calculate the second variation we first determine the derivative of our functional at an arbitrary point. Let $\left(d^{2} /(d t)^{2}\right)\left(J_{T_{t}}\right)=J^{\prime \prime}$. Then 


$$
\begin{aligned}
& \frac{d}{d t} I\left(\rho(., t), T_{t}\right) \\
&=(\varkappa-1) p_{0} \int_{B_{R}} \frac{d}{d t}\left(J_{T_{t}}\right) d x+\int_{B_{R}} \varkappa \rho^{\varkappa-1} \varphi J_{T_{t}}^{-\varkappa+1}+\rho^{\varkappa}(1-\varkappa) J_{T_{t}}^{-\varkappa} \frac{d}{d t}\left(J_{T_{t}}\right) d x \\
& \quad-\frac{A}{2 \pi} \int_{B_{R}} \int_{B_{R}} \frac{\varphi(x) \rho(y, t)}{\left|T_{t}(x)-T_{t}(y)\right|} d x d y \\
& \quad+\frac{A}{4 \pi} \int_{B_{R}} \int_{B_{R}} \frac{\rho(x, t) \rho(y, t)}{\left|T_{t}(x)-T_{t}(y)\right|^{3}}(u(x)-u(y)) \cdot\left(T_{t}(x)-T_{t}(y)\right) d x d y .
\end{aligned}
$$

This gives us the following result:

\section{Lemma 7.}

$$
V_{2}\left(\rho_{0}, u, \varphi\right)=\frac{d^{2}}{(d t)^{2}} I\left(\rho(., t), T_{t}\right)=E_{1}+E_{2}+E_{3},
$$

with

$$
\begin{aligned}
& E_{1}=(\varkappa-1) \int_{B_{R}} J^{\prime \prime}\left(p_{0}-\rho_{0}^{\varkappa}\right) d x \\
&+\varkappa(\varkappa-1) \int_{B_{R}} \rho_{0}^{\varkappa-2} \varphi^{2}-2 \rho_{0}^{\varkappa-1} \varphi \operatorname{div}(u)+\rho_{0}^{\varkappa}(\operatorname{div}(u))^{2} d x, \\
& E_{2}=\frac{A}{\pi} \int_{B_{R}} \int_{B_{R}} \frac{\rho_{0}(x) \varphi(y)}{|x-y|^{3}}(x-y) \cdot(u(x)-u(y)) d x d y \\
& \quad-\frac{A}{2 \pi} \int_{B_{R}} \int_{B_{R}} \frac{\varphi(x) \varphi(y)}{|x-y|} d x d y, \\
& E_{3}=\frac{A}{4 \pi} \int_{B_{R}} \int_{B_{R}} \frac{\rho_{0}(x) \rho_{0}(y)}{|x-y|^{3}}|u(x)-u(y)|^{2} d x d y \\
& \quad-\frac{3 A}{4 \pi} \int_{B_{R}} \int_{B_{R}} \frac{\rho_{0}(x) \rho_{0}(y)}{|x-y|^{5}}((x-y) \cdot(u(x)-u(y)))^{2} d x d y .
\end{aligned}
$$

First let us consider $E_{1}$. 
Lemma 8. Under our general assumptions we obtain the identity

$$
\begin{aligned}
E_{1}=\varkappa(\varkappa-1) & \int_{B_{R}} \rho_{0}^{\varkappa-2}\left(\varphi-\operatorname{div}\left(\rho_{0} u\right)\right)^{2} \\
& -\rho_{0}^{\varkappa-2}\left[\rho_{0}(u \cdot \nabla u) \cdot \nabla \rho_{0}+u \cdot \nabla \rho_{0} \operatorname{div}\left(\rho_{0} u\right)\right] \\
& +2 \rho_{0}^{\varkappa-2} \varphi u \cdot \nabla \rho_{0} d x .
\end{aligned}
$$

Proof. We have

$$
\begin{aligned}
\int_{B_{R}} J^{\prime \prime}\left(p_{0}-\rho_{0}^{\varkappa}\right) d x= & \int_{B_{R}} \operatorname{div}(\operatorname{div}(u) u-(u \cdot \nabla u))\left(p_{0}-\rho_{0}^{\varkappa}\right) d x \\
= & -\int_{B_{R}}(\operatorname{div}(u) u-(u \cdot \nabla u)) \cdot \nabla\left(p_{0}-\rho_{0}^{\varkappa}\right) d x \\
& \quad+\int_{\partial B_{R}}(\operatorname{div}(u) u-(u \cdot \nabla u)) \cdot n\left(p_{0}-\rho_{0}^{\varkappa}\right) d \sigma_{x} .
\end{aligned}
$$

Now as we assumed $u(x)=0$ or $\left(p_{0}-\rho_{0}^{\varkappa}\right)(x)=0$ on $\partial B_{R}$, the second integral vanishes, and we get

$$
\int_{B_{R}} J^{\prime \prime}\left(p_{0}-\rho_{0}^{\varkappa}\right) d x=\varkappa \int_{B_{R}} \rho_{0}^{\varkappa-1}(\operatorname{div}(u) u-(u \cdot \nabla u)) \cdot \nabla\left(\rho_{0}\right) d x .
$$

The remainder is obtained by straightforward calculation.

Lemma 9. We can write $E_{3}=E_{31}+E_{32}$ with

$$
\begin{aligned}
E_{31}=-\sum_{i, j=1}^{3} \int_{B_{R}} \rho_{0}(y) u_{i} u_{j} \frac{\partial^{2}}{\partial y_{j} \partial y_{i}}\left(\varkappa \rho_{0}^{\varkappa-1}(y)\right) d y, \quad \text { and } \\
\frac{2 \pi}{A} E_{32}=-\int_{B_{R}} \int_{B_{R}}\left(\operatorname{div}_{x}\left(\rho_{0} u(x)\right) \operatorname{div}_{y}\left(\rho_{0} u(y)\right) \frac{1}{|x-y|}\right) d x d y \\
\quad-\int_{\partial B_{R}} \int_{\partial B_{R}}(u \cdot n)(x)(u \cdot n)(y) \rho_{0}(x) \rho_{0}(y) \frac{1}{|x-y|} d \sigma_{x} d \sigma_{y} \\
+2 \int_{B_{R}} \int_{\partial B_{R}} \operatorname{div}_{x}\left(\rho_{0} u\right) \rho_{0}(y) u(y) \cdot n \frac{1}{|x-y|} d x d \sigma_{y} .
\end{aligned}
$$


Proof. By rather straightforward calculations we obtain

$$
\begin{aligned}
& \int_{B_{R}} \int_{B_{R}}-3 \frac{\rho_{0}(x) \rho_{0}(y)}{|x-y|^{5}}((x-y) \cdot(u(x)-u(y)))^{2} \\
& +\frac{\rho_{0}(x) \rho_{0}(y)}{|x-y|^{3}}|u(x)-u(y)|^{2} d x d y \\
& =6 \int_{B_{R}} \int_{B_{R}} \frac{\rho_{0}(x) \rho_{0}(y)}{|x-y|^{5}}(x-y) \cdot(u(x)-u(y))(x-y) \cdot u(y) d x d y \\
& -2 \int_{B_{R}} \int_{B_{R}} \frac{\rho_{0}(x) \rho_{0}(y)}{|x-y|^{3}}(u(x)-u(y)) \cdot u(y) d x d y \\
& =2 \int_{B_{R}} \int_{B_{R}} \rho_{0}(x) \rho_{0}(y)(x-y) \cdot(u(x)-u(y)) u(y) \cdot \nabla_{y}\left(\frac{1}{|x-y|^{3}}\right) d x d y \\
& -2 \int_{B_{R}} \int_{B_{R}} \frac{\rho_{0}(x) \rho_{0}(y)}{|x-y|^{3}}(u(x)-u(y)) \cdot u(y) d x d y \\
& =2 \sum_{i, j=1}^{3} \int_{B_{R}} \int_{B_{R}} \rho_{0}(x) \rho_{0}(y)\left(u_{i}(x)-u_{i}(y)\right) \\
& \times\left(-\frac{u_{i}(y)}{|x-y|^{3}} \delta_{i j}+\left(x_{i}-y_{i}\right) u_{j}(y) \frac{\partial}{\partial y_{j}}\left(\frac{1}{|x-y|^{3}}\right)\right) d x d y \\
& =2 \sum_{i, j=1}^{3} \int_{B_{R}} \int_{B_{R}} \rho_{0}(x) \rho_{0}(y)\left(u_{i}(x)-u_{i}(y)\right) u_{j}(y) \frac{\partial}{\partial y_{j}}\left(\frac{x_{i}-y_{i}}{|x-y|^{3}}\right) d x d y \\
& =J_{1} \text {. }
\end{aligned}
$$

From now until further notice the integrals denote Cauchy principal values. We can continue our chain of equations by

$$
\begin{aligned}
J_{1}=-2 & \sum_{i, j=1}^{3} \int_{B_{R}} \int_{B_{R}} \rho_{0}(x) \rho_{0}(y) u_{i}(y) u_{j}(y) \frac{\partial^{2}}{\partial y_{j} \partial y_{i}}\left(\frac{1}{|x-y|}\right) d x d y \\
& +2 \sum_{i, j=1}^{3} \int_{B_{R}} \int_{B_{R}} \rho_{0}(x) \rho_{0}(y) u_{i}(x) u_{j}(y) \frac{\partial^{2}}{\partial y_{j} \partial y_{i}}\left(\frac{1}{|x-y|}\right) d x d y=
\end{aligned}
$$




$$
=I_{1}+I_{2} .
$$

For the integral $I_{1}$ note

$$
\begin{aligned}
\int_{B_{R}} \rho_{0}(x) \frac{\partial^{2}}{\partial y_{j} \partial y_{i}}\left(\frac{1}{|x-y|}\right) d x & =\frac{\partial^{2}}{\partial y_{j} \partial y_{i}}\left(\int_{B_{R}} \rho_{0}(x) \frac{1}{|x-y|} d x\right)+\delta_{i j} \frac{4 \pi}{3} \rho_{0}(y) \\
& =\frac{\partial^{2}}{\partial y_{j} \partial y_{i}}\left(\frac{2 \pi \varkappa}{A} \rho_{0}^{\varkappa-1}(y)\right)+\delta_{i j} \frac{4 \pi}{3} \rho_{0}(y) .
\end{aligned}
$$

(See, e.g., Satz 12.5.3 (p. 226) in [16], or eq. (14) on p. 236, Chapter 11 Section 6 of [17], which unfortunately contains a misprint, as becomes clear by comparing with (12').) Therefore,

$$
I_{1}=-2 \int_{B_{R}} \rho_{0}(y) \sum_{i, j=1}^{3}\left[\frac{\partial^{2}}{\partial y_{j} \partial y_{i}}\left(\frac{2 \pi \varkappa}{A} \rho_{0}^{\varkappa-1}(y)\right) u_{i} u_{j}\right]+\frac{4 \pi}{3} \rho_{0}^{2}(y)|u|^{2} d y .
$$

Also,

$$
\begin{array}{r}
I_{2}=-2 \sum_{i, j=1}^{3} \int_{B_{R}} \int_{B_{R}} \frac{\partial}{\partial x_{i}}\left(\rho_{0}(x) \rho_{0}(y) u_{i}(x) u_{j}(y) \frac{\partial}{\partial y_{j}} \frac{1}{|x-y|}\right) d x d y \\
+2 \sum_{j=1}^{3} \int_{B_{R}} \int_{B_{R}} \operatorname{div}_{x}\left(\rho_{0} u(x)\right) \rho_{0}(y) u_{j}(y) \frac{\partial}{\partial y_{j}} \frac{1}{|x-y|} d x d y .
\end{array}
$$

The first expression is still a Cauchy principal value, and it equals

$$
\begin{aligned}
&-2 \sum_{i, j=1}^{3} \int_{B_{R}} \int_{\partial B_{R}} \frac{x_{i}}{|x|}\left(\rho_{0}(x) \rho_{0}(y) u_{i}(x) u_{j}(y) \frac{\partial}{\partial y_{j}} \frac{1}{|x-y|}\right) d \sigma_{x} d y \\
&+2 \lim _{\varepsilon \longrightarrow 0} \sum_{i, j=1}^{3} \int_{B_{R}} \int_{\partial\{|x-y|=\varepsilon\}} \frac{x_{i}-y_{i}}{|x-y|} \\
& \times\left(\rho_{0}(x) \rho_{0}(y) u_{i}(x) u_{j}(y) \frac{\partial}{\partial y_{j}} \frac{1}{|x-y|}\right) d \sigma_{x} d y .
\end{aligned}
$$

The second summand in the above sum equals

$$
\begin{aligned}
& 2 \lim _{\varepsilon \longrightarrow 0} \sum_{i, j=1}^{3} \int_{B_{R}} \int_{\partial\{|x-y|=\varepsilon\}} \frac{x_{i}-y_{i}}{|x-y|} \rho_{0}(x) \rho_{0}(y) u_{i}(x) u_{j}(y) \frac{x_{j}-y_{j}}{|x-y|^{3}} d \sigma_{x} d y \\
= & 2 \frac{4 \pi}{3} \int_{B_{R}}\left(\rho_{0}(y)\right)^{2}|u|^{2} d y .
\end{aligned}
$$


This cancels with the negative of the same quantity contained in $I_{1}$. In any case

$$
\begin{aligned}
I_{2}=-2 & \int_{\partial B_{R}} \int_{B_{R}}(u \cdot n)(x) \rho_{0}(x) \rho_{0}(y) u(y) \cdot \nabla_{y} \frac{1}{|x-y|} d y d \sigma_{x} \\
& +\frac{8 \pi}{3} \int_{B_{R}}\left(\rho_{0}(y)\right)^{2}|u|^{2} d y \\
& +2 \int_{B_{R}} \int_{B_{R}}\left(\operatorname{div}_{x}\left(\rho_{0} u(x)\right) \rho_{0}(y) u(y) \cdot \nabla_{y} \frac{1}{|x-y|}\right) d x d y .
\end{aligned}
$$

These are now all ordinary integrals again. Using the Divergence Theorem again, we obtain

$$
\begin{aligned}
I_{2}=- & 2 \int_{\partial B_{R}} \int_{\partial B_{R}}(u \cdot n)(x) \rho_{0}(x) \rho_{0}(y)(u \cdot n)(y) \frac{1}{|x-y|} d \sigma_{y} d \sigma_{x} \\
& -2 \int_{B_{R}} \int_{B_{R}} \operatorname{div}_{x}\left(\rho_{0} u\right) \operatorname{div}_{y}\left(\rho_{0} u\right) \frac{1}{|x-y|} d x d y \\
& +\frac{8 \pi}{3} \int_{B_{R}}\left(\rho_{0}(y)\right)^{2}|u|^{2} d y \\
& +4 \int_{B_{R}} \int_{\partial B_{R}} \operatorname{div}_{x}\left(\rho_{0} u\right) \rho_{0}(y) u(y) \cdot n \frac{1}{|x-y|} d x d \sigma_{y} .
\end{aligned}
$$

Putting these results together, we obtain our claim.

Lemma 10. We have $E_{2}=E_{21}+E_{22}$ with

$$
E_{21}=-2 \int_{B_{R}} \varphi(y) u(y) \cdot \nabla\left(\varkappa \rho_{0}^{\varkappa-1}(y)\right) d y
$$

and

$$
\begin{aligned}
\frac{2 \pi}{A} E_{22}=-\int_{B_{R}} & \int_{B_{R}} \frac{\varphi(x) \varphi(y)}{|x-y|} d x d y \\
& -2 \int_{B_{R}} \varphi(y) \int_{\partial B_{R}} \rho_{0}(R) u(x) \cdot n \frac{1}{|x-y|} d \sigma_{x} d y \\
& +2 \int_{B_{R}} \varphi(y) \int_{B_{R}} \operatorname{div}_{x}\left(\rho_{0} u\right)(x) \frac{1}{|x-y|} d x d y
\end{aligned}
$$


Proof.

$$
\begin{aligned}
& \int_{B_{R}} \int_{B_{R}} \frac{\rho_{0}(x) \varphi(y)}{|x-y|^{3}}(x-y) \cdot(u(x)-u(y)) d x d y \\
& =\int_{B_{R}} \int_{B_{R}} \frac{\rho_{0}(x) \varphi(y)}{|x-y|^{3}}(x-y) \cdot u(x) d x d y \\
& -\int_{B_{R}} \int_{B_{R}} \frac{\rho_{0}(x) \varphi(y)}{|x-y|^{3}}(x-y) \cdot u(y) d x d y \\
& =-\int_{B_{R}} \varphi(y) \int_{B_{R}} \rho_{0}(x) u(x) \cdot \nabla_{x} \frac{1}{|x-y|} d x d y \\
& -\int_{B_{R}}(\varphi u)(y) \cdot \int_{B_{R}} \rho_{0}(x) \nabla_{y} \frac{1}{|x-y|} d x d y \\
& =\int_{B_{R}} \varphi(y) \int_{B_{R}} \operatorname{div}_{x}\left(\rho_{0}(x) u(x)\right) \frac{1}{|x-y|} d x d y \\
& -\int_{B_{R}} \varphi(y) \int_{B_{R}} \operatorname{div}_{x}\left(\frac{\rho_{0}(x) u(x)}{|x-y|}\right) d x d y \\
& -\int_{B_{R}} \varphi(y) u(y) \cdot \nabla_{y}\left(\int_{B_{R}} \frac{\rho_{0}(x)}{|x-y|} d x\right) d y \\
& =-\int_{B_{R}} \varphi(y) \int_{\partial B_{R}} \rho_{0}(R) u(x) \cdot n \frac{1}{|x-y|} d \sigma_{x} d y \\
& +\int_{B_{R}} \varphi(y) \int_{B_{R}} \operatorname{div}_{x}\left(\rho_{0} u\right)(x) \frac{1}{|x-y|} d x d y \\
& -\int_{B_{R}} \varphi(y) u(y) \cdot \nabla\left(\frac{2 \pi \varkappa}{A} \rho_{0}^{\varkappa-1}(y)\right) d y .
\end{aligned}
$$

Now we obtain the following result:

Lemma 11. We have

$$
\begin{aligned}
E_{1}+E_{21}+E_{31}=-\varkappa & (\varkappa-1) \rho_{0}^{\varkappa-1}(R) \rho_{0}^{\prime}(R) \int_{\partial B_{R}}(u \cdot n)^{2} d \sigma_{x} \\
& +\varkappa(\varkappa-1) \int_{B_{R}} \rho_{0}^{\varkappa-2}\left(\operatorname{div}\left(\rho_{0} u\right)-\varphi\right)^{2} d x .
\end{aligned}
$$


Proof. First observe that

$$
\begin{aligned}
E_{1}+E_{21}=\varkappa(\varkappa-1) & \int_{B_{R}} \rho_{0}^{\varkappa-2}\left(\varphi-\operatorname{div}\left(\rho_{0} u\right)\right)^{2} \\
& -\rho_{0}^{\varkappa-2}\left[\rho_{0}(u \cdot \nabla u) \cdot \nabla \rho_{0}+u \cdot \nabla \rho_{0} \operatorname{div}\left(\rho_{0} u\right)\right] d x .
\end{aligned}
$$

In addition we have, integrating by parts

$$
\begin{gathered}
E_{31}=-\sum_{i, j=1}^{3} \int_{B_{R}} \rho_{0}(x) u_{i} u_{j} \frac{\partial^{2}}{\partial x_{j} \partial x_{i}}\left(\varkappa \rho_{0}^{\varkappa-1}(x)\right) d x \\
=-\int_{\partial B_{R}} \varkappa \rho_{0}(u \cdot n)(u \cdot \nabla) \rho_{0}^{\varkappa-1} d \sigma_{x} \\
+\sum_{i, j=1}^{3} \int_{B_{R}} \varkappa \frac{\partial}{\partial x_{i}}\left(\rho_{0} u_{i} u_{j}\right) \frac{\partial}{\partial x_{j}} \rho_{0}^{\varkappa-1} d x \\
=-\varkappa(\varkappa-1) \rho_{0}^{\varkappa-1}(R) \rho_{0}^{\prime}(R) \int_{\partial B_{R}}(u \cdot n)^{2} d \sigma_{x} \\
+\quad \int_{B_{R}} \operatorname{div}\left(\rho_{0} u\right)(u \cdot \nabla) \rho_{0}^{\varkappa-1} d x \\
+\int_{B_{R}} \varkappa \rho_{0}((u \cdot \nabla u) \cdot \nabla) \rho_{0}^{\varkappa-1} d x .
\end{gathered}
$$

From this our claim becomes obvious.

Now we introduce the notation $\alpha(x)=\varphi-\operatorname{div}\left(\rho_{0} u\right)$. Then we have the following result:

Lemma 12. For the remaining expressions we obtain

$$
\begin{aligned}
\frac{2 \pi}{A}\left(E_{22}+E_{32}\right)=-2 \rho_{0} & (R) \int_{B_{R}} \int_{\partial B_{R}} \frac{\alpha(y) u \cdot n(x)}{|x-y|} d \sigma_{x} d y \\
& -\rho_{0}^{2}(R) \int_{\partial B_{R}} \int_{\partial B_{R}} \frac{(u \cdot n)(x)(u \cdot n)(y)}{|x-y|} d \sigma_{x} d \sigma_{y} \\
& -\int_{B_{R}} \int_{B_{R}} \frac{\alpha(x) \alpha(y)}{|x-y|} d x d y .
\end{aligned}
$$

Proof. This is easy to check by elementary calculation, reducing the necessary regularity of $u$ to $C^{1}$ by approximation. 
Now we can give our modified form of the second variation.

Theorem 2. If $u \in C^{1}\left(\bar{B}_{R}\right)$ and $\varphi \in C^{0}\left(\bar{B}_{R}\right)$, then with $\alpha(x)=$ $\varphi-\operatorname{div}\left(\rho_{0} u\right)$ we have

$$
\begin{aligned}
V_{2}\left(\rho_{0}, u, \varphi\right)=\varkappa(\varkappa & -1) \int_{B_{R}} \rho_{0}^{\varkappa-2} \alpha^{2} d x-\frac{A}{2 \pi} \int_{B_{R}} \int_{B_{R}} \frac{\alpha(x) \alpha(y)}{|x-y|} d x d y \\
& -\varkappa(\varkappa-1) \rho_{0}^{\varkappa-1}(R) \rho_{0}^{\prime}(R) \int_{\partial B_{R}}(u \cdot n)^{2} d \sigma_{x} \\
& -\frac{A \rho_{0}(R)}{\pi} \int_{B_{R}} \alpha(y) \int_{\partial B_{R}} \frac{u \cdot n(x)}{|x-y|} d \sigma_{x} d y \\
& -\frac{A \rho_{0}^{2}(R)}{2 \pi} \int_{\partial B_{R}} \int_{\partial B_{R}} \frac{(u \cdot n)(x)(u \cdot n)(y)}{|x-y|} d \sigma_{x} d \sigma_{y} .
\end{aligned}
$$

Now notice that $V_{2}$ only depends on $u \cdot n \mid \partial B_{R}$, and $\varphi-\operatorname{div}\left(\rho_{0} u\right)$. Any two combinations of variations of the domain and the density for which these two quantities are the same will lead to the same value. In the final chapter we will use spherical harmonics to move a little further with our analysis. To this end we make the following definition:

Definition 2. For $\alpha \in C^{0}\left(\bar{B}_{R}\right)$ and $f \in C^{1}\left(S^{2}\right)$ with

$$
-\int_{B_{R}} \alpha d x=R \rho_{0}(R) \int_{\partial B_{R}} f\left(\frac{x}{|x|}\right) d \sigma
$$

let $\widehat{V}_{2}\left(\rho_{0}, \alpha, f\right)=V_{2}\left(\rho_{0}, u, \varphi\right)$ for $u(x)=R^{-4}|x|^{4} f(x /|x|) x$ and $\varphi=\alpha+$ $\operatorname{div}\left(\rho_{0} u\right)$.

Note that on $\partial B_{R}$ we have $u(x) \cdot n=R f(x / R)$, and that the equation (7) is an expression of

$$
\int_{B_{R}} \varphi d x=0
$$

the restriction that the mass is to remain constant during the variation.

\section{The Lane-Emden Equation}

In this section we consider the problem of finding a spherically symmetric configuration of minimal energy described by its density $\rho(r)$. We will see that $s(r)=\rho^{\varkappa-1}(r)$ has to be a so-called $E$-solution (see, e.g. [5]) of the LaneEmden equation (4)

$$
s^{\prime \prime}(r)+\frac{2}{r} s^{\prime}(r)=-\frac{2 A}{\varkappa}(s(r))^{1 /(\varkappa-1)} .
$$


Such solutions are characterized by the fact that $s(r) \rightarrow a>0$ as $r \rightarrow 0$. This equation has been studied extensively in the literature, and we will be able to use many of the known results. As we consider a problem with a non-zero outside pressure we will also need to derive some new ones. The following theorem contains a combination of known results about this equation.

Theorem 3. Given $s_{0}>0$ there is an $R<\infty$ and an E-solution $s \in$ $C^{\infty}([0, R])$ of (4) with $s(0)=s_{0}, s(r)>0$ for $r>R$, and $s(R)=0$. Any solution $\widehat{s}$ of (4) on an interval $\left(0, r_{1}\right) \subset[0, R]$ with $\lim _{r \rightarrow 0} \widehat{s}(r)=s_{0}$ must be identical to $s$ where it exists.

Proof. Existence and uniqueness are an easy consequence of Theorem 3.6 in [2], together with Theorem 3 in [5], which assures that $R$ is finite. The regularity at $r=0$ can be obtained by using the fact that the solution actually solves a Poisson problem in three dimensions.

For the remainder of this section let

$$
\mu=\frac{1}{2}\left(\frac{2-\varkappa}{\varkappa-1}\right) .
$$

We continue the functions $s$ we just obtained as zero on $[R, \infty)$ to get $s \in$ $C^{0}([0, \infty))$. Note that $s$ are all decreasing functions, and even strictly decreasing where they are positive. Now let $\Psi:[0, \infty) \rightarrow \mathbb{R}$ be the $s$ belonging to $s_{0}=1$. Due to the scaling properties of (4), which are easy to verify explicitly and are contained, e.g., in Lemma 3 in [5] we have that $s(r)=s_{0} \Psi\left(s_{0}^{\mu} r\right)$.

This means that all the functions $s$ belong to a continuous one-parameterfamily of solutions. Now for $s_{0}, R, \rho>0$ let

$$
\begin{aligned}
& \mathcal{M}_{1}\left(s_{0}, R\right)=4 \pi \int_{0}^{R}\left(s_{0} \Psi\left(s_{0}^{\mu} r\right)\right)^{1 /(\varkappa-1)} r^{2} d r, \quad \text { and } \\
& \mathcal{M}_{2}\left(s_{0}, \rho\right)=4 \pi s_{0}^{1 /(\varkappa-1)-3 \mu} \int_{0}^{\rho}(\Psi(\xi))^{1 /(\varkappa-1)} \xi^{2} d \xi .
\end{aligned}
$$

$\mathcal{M}_{1}$ gives the mass enclosed in the ball of radius $R$ by the mass distribution $s_{0} \Psi\left(s_{0}^{\mu} r\right)$. We can prove the following result:

Lemma 13. The functions $\mathcal{M}_{1}$ and $\mathcal{M}_{2}$ belong to $C^{1}\left((0, \infty)^{2}\right)$ and we have $\mathcal{M}_{1}\left(s_{0}, R\right)=\mathcal{M}_{2}\left(s_{0}, s_{0}^{\mu} R\right), \frac{\partial \mathcal{M}_{1}}{\partial s_{0}}>0, \quad \frac{\partial \mathcal{M}_{1}}{\partial R} \geq 0, \quad \frac{\partial \mathcal{M}_{2}}{\partial s_{0}}>0, \quad \frac{\partial \mathcal{M}_{2}}{\partial \rho} \geq 0$.

The inequality for $\mathcal{M}_{1}$ is strict where $\Psi\left(s_{0}^{\mu} R\right)>0$, that for $\mathcal{M}_{2}$ is strict where $\Psi(\rho)>0$. In addition $\lim _{s_{0} \rightarrow \infty} \mathcal{M}_{1}\left(s_{0}, R\right)=\infty$ for $R>0$. 
Proof. The claimed identity is easily proved by the substitution $\xi=s_{0}^{\mu} r$ in the integral. The existence and continuity of the partial derivatives is obvious for $\mathcal{M}_{2}$, and immediately follows for $\mathcal{M}_{1}$ by means of the identity above. The inequalities are also clear for $\mathcal{M}_{2}$ as $1 /(\varkappa-1)-3 \mu>0$. For $\mu \geq 0$, i.e. $\varkappa \leq 2$, they also immediately follow for $\mathcal{M}_{2}$ from those for $\mathcal{M}_{1}$, and the statement about the limit is easy to see as well. For $\varkappa>2$ we have $\mu<0$, so $\Psi\left(s_{0}^{\mu} r\right)$ is an increasing function of $s_{0}$, which shows both the positivity of the derivative and the fact that the limit is infinite.

Lemma 14. Let $M>0$ be given. For every $R>0$ there is exactly one number $s_{0}>0$ such that $\mathcal{M}_{1}\left(s_{0}, R\right)=M$. The function $S_{1}=S_{1}(R)$ assigning this $s_{0}$ to $R$ belongs to $C^{1}((0, \infty))$. There is a radius $R_{1}$ only depending on $M$ such that $\Psi\left(S_{1}^{\mu}(R) R\right)>0$ for $R<R_{1}$ and $\Psi\left(S_{1}^{\mu}(R) r\right)=0$ for $r \in\left[R_{1}, R\right]$. The function $S_{1}$ also belongs to $C^{3}\left(\left(0, R_{1}\right) \cup\left(R_{1}, \infty\right)\right)$, and for $R<R_{1}$ we have $S_{1}^{\prime}(R)<0$, while $S_{1}^{\prime}(R)=0$ for $R \geq R_{1}$. Let $\mathfrak{D}(R)=S_{1}(R) \Psi\left(S_{1}^{\mu}(R) R\right)$. For $R<R_{1}$ we have $\mathfrak{D}^{\prime}(R)<0$, and $\mathfrak{D}(R)=0$ for $R>R_{1}$, finally $\mathfrak{D}(R) \rightarrow \infty$ as $R \rightarrow 0$.

Proof. The existence and uniqueness of $s_{0}$ immediately follows from Lemma 13. As $\partial \mathcal{M}_{1} / \partial s_{0} \neq 0$, the implicit function theorem implies $S_{1} \in C^{1}((0, \infty))$. Also, as $\mathcal{M}_{1}\left(S_{1}(R), R\right)=M$ we have

$$
\frac{\partial \mathcal{M}_{1}}{\partial s_{0}}\left(S_{1}(R), R\right) S_{1}^{\prime}(R)+\frac{\partial \mathcal{M}_{1}}{\partial R}\left(S_{1}(R), R\right)=0,
$$

so $S_{1}^{\prime}(R) \leq 0$, and $<0$ if $\Psi\left(S_{1}^{\mu}(R) R\right)>0$. With $g(R)=S_{1}^{\mu}(R) R$ we have $\mathcal{M}_{2}\left(S_{1}(R), g(R)\right)=M$ and therefore

$$
\frac{\partial \mathcal{M}_{2}}{\partial s_{0}}\left(S_{1}(R), g(R)\right) S_{1}^{\prime}(R)+\frac{\partial \mathcal{M}_{2}}{\partial \rho}\left(S_{1}(R), g(R)\right) g^{\prime}(R)=0,
$$

so $g^{\prime}(R)>0$ if $\Psi\left(S_{1}^{\mu}(R) R\right)>0$. This implies $(\Psi \circ g)^{\prime}(R)<0$ and $\mathfrak{D}^{\prime}(R)<0$ if $\Psi(g(R))>0$, as $\Psi$ is decreasing. So $\Psi \circ g$ is decreasing where it is positive, and therefore has to stay zero once it becomes zero. Let $R_{1}$ be the smallest number for which it equals zero. It is clear it has the properties we claimed. If the convergence to infinity at zero would not take place, the solutions would be bounded as $R \rightarrow 0$, which would make the mass go to zero. Now we have proved all our claims.

Lemma 15. Assume $M>0$ is given. For every $p_{0} \geq 0$ there is exactly one $R>0$ and one $s_{0}>0$ such that $\mathcal{M}_{1}\left(s_{0}, R\right)=M$ and $\left(s_{0} \Psi\left(s_{0}^{\mu} r\right)\right)^{\varkappa /(\varkappa-1)}=p_{0}$, while $s_{0} \Psi\left(s_{0}^{\mu} r\right)>0$ for $r<R$. The function $S_{2}=S_{2}\left(p_{0}\right)$ assigning $s_{0}$ to $p_{0}$ belongs to $C^{2}((0, \infty))$. 
Proof. As prescribing $p_{0}>0$ is equivalent to prescribing $s(R)$ for a solution of given mass, it is equivalent to solving $\mathfrak{D}(R)=c$, and that is obviously possible in the way claimed by the previous Lemma. The uniqueness for $p_{0}=0$ follows from eqs. (7.3) and (7.4) in [3].

Now we want to show that for each of these problems there are density distributions that minimize energy and fulfill the Euler equations, so by what we have proved the unique solutions of the Euler equations must be the minimizers. The next few lemmas can more easily be proved for a more general case than the one to be used here, and so we are doing just that. Notice in particular that Lemmas $16,17,18$, and 19 are also true for $p_{0}=0$. First we have to find a simple expression for the energy of radially symmetric mass distributions.

Lemma 16. Let $R>0$ and $\rho \in C^{0}([0, R])$. Then we have

$$
\begin{aligned}
& E\left(\rho(|x|), B_{R}\right) \\
= & 4 \pi\left[\int_{0}^{R}\left((\varkappa-1) p_{0}+\rho^{\varkappa}(r)\right) r^{2} d r-A \int_{0}^{R} \int_{0}^{R} \min \left(r^{2} s, s^{2} r\right) \rho(r) \rho(s) d r d s\right] .
\end{aligned}
$$

Proof. The claim is an easy consequence of Lemma 5, also keeping in mind that constants are spherical harmonics of order zero.

To continue we need a few definitions.

Definition 3. For any bounded open set $\Omega \subset \mathbb{R}^{3}$ let

$$
\begin{gathered}
A(\Omega, M)=\left\{\rho \in L_{\varkappa}(\Omega): \int_{\Omega} \rho d x=M\right\}, \\
\widehat{A}\left(B_{R}, M\right)=\left\{\rho \in A\left(B_{R}, M\right): \rho(x)=\rho(O x) \text { for any orthogonal } O\right\}, \text { and } \\
e(R)=\inf _{\rho \in \hat{A}\left(B_{R}, M\right)} E\left(\rho, B_{R}\right) .
\end{gathered}
$$

Then we have the following result:

Lemma 17. Let $\Omega \subset \mathbb{R}^{3}$ be an open bounded set. Let $M<\infty$ and $\varkappa>\frac{4}{3}$. There is a number $C_{1}>0$ depending on $M$, and independent of $\Omega$ such that for all $\rho \geq 0$ with

$$
\int_{\Omega} \rho(x) d x=M
$$


we have even with $p_{0}=0$ that

$$
E(\rho, \Omega) \geq-C_{1}+\frac{1}{2}\|\rho\|_{L_{\varkappa}}^{\varkappa} \geq-C_{1}+\frac{M^{\varkappa}}{2(m(\Omega))^{\varkappa-1}} .
$$

Proof. For $\alpha \in(1, \varkappa)$, we have

$$
\|\rho\|_{L_{\alpha}}^{\alpha} \leq C\|\rho\|_{L_{\varkappa}}^{(\alpha-1)(\varkappa /(\varkappa-1))}\|\rho\|_{L_{1}}^{(\varkappa-\alpha) /(\varkappa-1)} .
$$

Therefore

$$
\|\rho\|_{L_{\alpha}} \leq C\|\rho\|_{L_{\varkappa}}^{((\alpha-1) / \alpha)(\varkappa /(\varkappa-1))}
$$

with a constant $C$ dependent on $M$ and $\alpha$. Introducing

$$
V(x)=\int_{\Omega} \frac{\rho(y)}{|x-y|} d y,
$$

we obtain by Calderòn-Zygmund and the Sobolev imbedding theorem (See [10], Theorem 7.10 and Theorem 9.9) that if $1 / \alpha_{2}=1 /(3 \varkappa)+\frac{2}{3}$, we have

$$
\|V\|_{L_{3 \varkappa}} \leq C\|\rho\|_{L_{\alpha_{2}}}
$$

for some constant $C$ as $1 /(3 \varkappa)=1 / \alpha_{2}-\frac{2}{3}$, so by the Hölder inequality we get

$$
\left|\int_{\Omega} V \rho d x\right| \leq\|V\|_{L_{3 \varkappa}}\|\rho\|_{L_{\alpha_{1}}} \leq C\|\rho\|_{L_{\alpha_{2}}}\|\rho\|_{L_{\alpha_{1}}}
$$

with $1 / \alpha_{1}+1 /(3 \varkappa)=1$, so $1 / \alpha_{1}>1-\frac{1}{4}=\frac{3}{4}$, so $\alpha_{1}<\frac{4}{3}$. Putting our results so far together, we get

$$
\begin{aligned}
\left|\int_{\Omega} V \rho d x\right| & \leq C\|\rho\|_{L_{\alpha_{2}}}\|\rho\|_{L_{\alpha_{1}}} \\
& \leq C\|\rho\|_{L_{\varkappa}}^{\left(\left(\alpha_{1}-1\right) / \alpha_{1}\right)(\varkappa /(\varkappa-1))}\|\rho\|_{L_{\varkappa}}^{\left(\left(\alpha_{2}-1\right) / \alpha_{2}\right)(\varkappa /(\varkappa-1))} \\
& =C\|\rho\|_{L_{\varkappa}}^{(\varkappa /(\varkappa-1))\left(\left(\alpha_{1}-1\right) / \alpha_{1}+\left(\alpha_{2}-1\right) / \alpha_{2}\right)} \\
& =C\|\rho\|_{L_{\varkappa}}^{(\varkappa /(\varkappa-1))\left(1-1 / \alpha_{1}+1-1 / \alpha_{2}\right)} \\
& =C\|\rho\|_{L_{\varkappa}}^{(\varkappa /(\varkappa-1))(2-5 / 3)} \\
& =C\|\rho\|_{L_{\varkappa}}^{(1 / 3)(\varkappa /(\varkappa-1))} .
\end{aligned}
$$

Now also $\frac{1}{3}(\varkappa /(\varkappa-1))<\varkappa$, so we have

$$
E(\rho, \Omega) \geq\|\rho\|_{L_{\varkappa}}^{\varkappa}-C\|\rho\|_{L_{\varkappa}}^{(1 / 3)(\varkappa /(\varkappa-1))} \geq-C_{1}+\frac{1}{2}\|\rho\|_{L_{\varkappa}}^{\varkappa}
$$


as we claimed. For the second inequality note

$$
\int_{\Omega} \rho(x) d x \leq\left(\int_{\Omega} 1^{\varkappa /(\varkappa-1)} d x\right)^{(\varkappa-1) / \varkappa}\|\rho\|_{L_{\varkappa}} .
$$

Lemma 18. If $\Omega$ is bounded, then in every weakly (in $L_{\varkappa}$ ) closed subclass of $A(\Omega, M)$ the functional $I(\rho, \Omega)$ has a minimum.

Proof. Consider any sequence $\rho_{k} \in A(\Omega, M)$ with $E\left(\rho_{k}, \Omega\right)$ converging to the infimum of this quantity over the set in question. We can select a subsequence weakly convergent in $L_{\varkappa}$ to a function $\widetilde{\rho}_{0}$. Now

$$
\int_{\Omega}(\varkappa-1) p_{0}+\rho^{\varkappa}(x) d x
$$

is of course lower semicontinuous with respect to weak convergence and

$$
\int_{\Omega} \int_{\Omega} \frac{\rho_{k}(x) \rho_{k}(y)}{|x-y|} d x d y=\int_{\Omega} \rho_{k}(x) V_{k}(x) d x .
$$

(see (11)) Now $V_{k}(x)$ converges strongly in $L_{p}$ for any $p<12$ by a combination of Calderòn-Zygmund and the Sobolev imbedding theorem, so the double integral converges as $k \rightarrow \infty$. This proves the minimizing character of $\widetilde{\rho}_{0}$.

Now we can prove the existence of a symmetric density distribution minimizing energy in its class.

Lemma 19. Let $M>0$ and $R>0$ be given. Then there is exactly one function $s:[0, R] \rightarrow \mathbb{R}, s \in C^{0}([0, R])$, with $s(r) \geq 0$ such that

$$
\rho_{0}(x)=s^{1 /(\varkappa-1)}(|x|)
$$

has the property that $\rho_{0} \in A\left(B_{R}, M\right)$ and

$$
E\left(\rho_{0}, B_{R}\right)=e(R) .
$$

There is an $R_{1}$ depending only on $M$ and $\varkappa$, such that $s(r)>0$ for $r<R_{1}$ and $s(r)=0$ for $r \geq R_{1}$, and $s \in C^{2}\left(\left[0, \min \left(R_{1}, R\right)\right]\right)$ fulfills $(4)$ in $\left(0, \min \left(R_{1}, R\right)\right]$.

Proof. Let $\rho_{0}(x)=\rho_{*}(|x|)$. As the set of spherically symmetric functions is weakly closed, we have by Lemma 18 that there is a minimizing function $\rho_{0}$ in $A\left(B_{R}, M\right)$. Now let $\varphi$ be such that $\varphi(|x|) \in L_{\varkappa}\left(B_{R}\right),|\varphi(r)| \leq \frac{1}{2} \rho_{*}(r)$ where $\rho_{*}(r)>0, \varphi(r) \geq 0$ where $\rho_{*}(r)=0$ and $t \in[0,1]$. Then it is easy to see by distinguishing the cases $\rho_{*}(r)=0$ and $\rho_{*}(r)>0$ that

$$
0 \leq \rho_{*}+t \varphi \text { and }\left(\rho_{*}+t \varphi\right)^{\varkappa} \leq \rho_{*}^{\varkappa}+t \varkappa \rho_{*}^{\varkappa-1} \varphi+\varkappa^{2} t^{\varkappa} 2^{\varkappa}\left(\rho_{*}^{\varkappa}+|\varphi|^{\varkappa}\right) \text {. }
$$


Using the notation,

$$
E(r)=r^{2} \varkappa \rho_{*}^{\varkappa-1}(r)-2 A \int_{0}^{R} \min \left(r^{2} \alpha, \alpha^{2} r\right) \rho_{*}(\alpha) d \alpha
$$

we obtain that

$$
\int_{0}^{R} E(r) \varphi(r) d r \geq 0 \text { if } \int_{0}^{R} \varphi(\alpha) \alpha^{2} d \alpha=0
$$

First use

$$
\varphi(r)= \pm \rho_{*}(r)\left[\phi(r)-\frac{4 \pi}{M} \int_{0}^{R} \rho_{*}(\alpha) \phi(\alpha) \alpha^{2} d \alpha\right]
$$

with $|\phi(r)| \leq \frac{1}{4}$. It always fulfills the integral condition, so we obtain

$$
\int_{0}^{R} E(r) \varphi(r) d r=0
$$

with this $\varphi$. This means

$$
\begin{aligned}
\int_{0}^{R} E(r) \rho_{*}(r) \phi(r) d r & =\int_{0}^{R} E(r) \rho_{*}(r) d r \frac{4 \pi}{M} \int_{0}^{R} \rho_{*}(\alpha) \phi(\alpha) \alpha^{2} d \alpha \\
& =-C \int_{0}^{R} \rho_{*}(\alpha) \phi(\alpha) \alpha^{2} d \alpha
\end{aligned}
$$

with a constant $C$ independent of $\phi$. This means

$$
\int_{0}^{R}\left[E(r)+r^{2} C\right] \rho_{*}(r) \phi(r) d r=0
$$

for all $\phi \in L_{\infty}(0, R)$. Therefore $E(r)=-r^{2} C$ or

$$
r^{2} \varkappa \rho_{*}^{\varkappa-1}(r)=2 A \int_{0}^{R} \min \left(r^{2} \alpha, \alpha^{2} r\right) \rho_{*}(\alpha) d \alpha-r^{2} C
$$

for almost all $r$ for which $\rho_{*}(r)>0$. Going back to a general $\varphi$ we also get that

$$
\int_{0}^{R}\left[r^{2} \varkappa \rho_{*}^{\varkappa-1}(r)-2 A \int_{0}^{R} \min \left(r^{2} \alpha, \alpha^{2} r\right) \rho_{*}(\alpha) d \alpha+C r^{2}\right] \varphi(r) d r \geq 0
$$

for all such admissible $\varphi$, and then the integral becomes entirely independent of the values of $\varphi$ on the set where $\rho_{*}(r)>0$, as the expression in the brackets vanishes there. So this integral is non-negative for all $\varphi$ which are non-negative 
where $\rho_{*}(r)=0$, and vanish elsewhere, as we can modify $\varphi$ in any way we want where $\rho_{*}(r)>0$ to fulfill the condition $\int_{0}^{R} \varphi(\alpha) \alpha^{2} d \alpha=0$. Thus

$$
r^{2} \varkappa \rho_{*}^{\varkappa-1}(r) \geq 2 A \int_{0}^{R} \min \left(r^{2} \alpha, \alpha^{2} r\right) \rho_{*}(\alpha) d \alpha-C r^{2}
$$

almost everywhere, and we have equality at almost every point where $\rho_{*}(r)>0$, which is by the inequality always true if its right side is positive. So we find that both sides even must be almost everywhere equal where one of them is positive, and we get, again up to measure zero,

$$
r^{2} \varkappa \rho_{*}^{\varkappa-1}(r)=\max \left(2 A \int_{0}^{R} \min \left(r^{2} \alpha, \alpha^{2} r\right) \rho_{*}(\alpha) d \alpha-C r^{2}, 0\right) .
$$

This immediately implies $\rho_{*}$ is continuous in $(0, R]$. Defining $s(r)=\rho_{*}^{\varkappa-1}(r)$ we get that where $s(r)>0$ we have

$$
s^{\prime}(r)=-\frac{2 A}{\varkappa r^{2}} \int_{0}^{r} \rho_{*}(\alpha) \alpha^{2} d \alpha .
$$

Thus $s(r)$ is decreasing where it is positive, and therefore, as $r$ increases, always stays zero where it is zero. Also by (12) we have $b=\lim _{r \rightarrow 0} r^{2} s^{\prime}(r)=0$. As it is decreasing as a function of $r$, we know $\lim _{r \downarrow 0} s(r)=a$ exists, and is either finite, in which case $s$ is an $E$-solution (see [5]), or infinite, in which case it is an $M$-solution. As $b=0$, the function $s$ cannot be an $M$-solution by Theorem 2 in [5], so it has to be an $E$-solution. So $s$ is bounded and we get from (12) that $s^{\prime}(r)$ is also bounded, and then using this equation again, we get $s^{\prime}(r) / r$ converges to a finite number. By the Lane-Emden equation itself we then get $s$ is twice continuously differentiable near $r=0$. By Lemma 14 already the fact that $s$ fulfills the equation where it is positive, and that it has a given mass, determines it uniquely.

Theorem 4. The function e belongs to $C^{1}(0, \infty)$, and goes to infinity as $R$ goes to zero or infinity. With the $R_{1}$ from Lemma 19 we have $e \in C^{2}\left(\left(0, R_{1}\right) \cup\right.$ $\left.\left(R_{1}, \infty\right)\right)$, and there is exactly one point $R_{0} \in\left(0, R_{1}\right)$ with $e^{\prime}\left(R_{0}\right)=0$, and there $e^{\prime \prime}\left(R_{0}\right)>0$ and the absolute minimum of $e$ is attained.

Proof. First we can see that $e$ is continuous, as the minimizing function depends continuously on $R$ by Lemma 14. The second inequality in Lemma 17 gives us $e(R) \rightarrow \infty$ as $R \rightarrow 0$. For $R \geq R_{1}$ the minimizing $s$ will be zero between $R_{1}$ and $R$ and otherwise be independent of $R$. This means that $e(R)=(\varkappa-1) p_{0} \frac{4}{3} \pi R^{3}+C$ there, and thus is smooth and goes to infinity as $R$ goes to infinity. From Lemma 14 we know that for $R<R_{1}$ the function $S_{1}(R)$ is 
three times differentiable. Therefore its energy $e(R)$ also belongs to $C^{3}\left(0, R_{1}\right)$. From Lemma 3 we can derive

$$
e^{\prime}(R)=4 \pi R^{2}(\varkappa-1)\left(p_{0}-\widehat{\rho}^{\varkappa}(R)\right),
$$

for $R_{1} \neq R$, where $\widehat{\rho}(R)$ is the density of the solution for $R$ at $r=R$, which we can easily see is given by $\widehat{\rho}(R)=(\mathfrak{D}(R))^{1 /(\varkappa-1)}$, and so

$$
\left(4 \pi R^{2}(\varkappa-1)\right)^{-1} e^{\prime}(R)=p_{0}-(\mathfrak{D}(R))^{\varkappa /(\varkappa-1)} .
$$

As $e(R)$ and the right side are continuous, $e$ also belongs to $C^{1}((0, \infty))$. By Lemma 14 the right side is weakly increasing on $(0, \infty)$, and strictly increasing for $R<R_{1}$. As $e$ is a continuous function on $(0, \infty)$ going to infinity at 0 and $\infty$, it must have a minimum for some $R=R_{0}$. Clearly $e^{\prime}(R)>0$ for $R \geq R_{1}$, and so $R_{0}<R_{1}$. As $e^{\prime}$ is strictly increasing in $\left(0, R_{1}\right)$ there can be only one $R_{0}$ with $e^{\prime}\left(R_{0}\right)=0$. Taking the derivative of (13) at $R_{0}$ then gives

$$
\left(4 \pi R_{0}^{2}(\varkappa-1)\right)^{-1} e^{\prime \prime}\left(R_{0}\right)=-\frac{\varkappa}{\varkappa-1}\left(\mathfrak{D}\left(R_{0}\right)\right)^{\varkappa /(\varkappa-1)-1} \mathfrak{D}^{\prime}\left(R_{0}\right)>0 .
$$

\section{Relative Minimality}

Now we can show the strict positivity of the second variation with respect to all variations which are not just translations. To this end we have to calculate the second variation in terms of functions given as expansions in spherical harmonics. To enable us to do this we define as follows.

Definition 4. Let

$$
\begin{aligned}
& B_{1}=\varkappa(\varkappa-1), \\
& B_{2}=4 A, \\
& B_{3}=4 A \rho_{0}(R) R^{2}, \\
& B_{4}=-\varkappa(\varkappa-1) R^{4} \rho_{0}^{\varkappa-1}(R) \rho_{0}^{\prime}(R), \\
& B_{5}=2 R^{5} A \rho_{0}^{2}(R) .
\end{aligned}
$$

Then for $h$ with $r h(r) \in L_{2}((0, R)), n$ a non-negative integer, and $a \in \mathbb{R}$ let

$$
\begin{aligned}
V_{2, n}\left(\rho_{0}, h, a\right)=B_{1} & \int_{0}^{R} \rho_{0}^{\varkappa-2} h^{2} r^{2} d r \\
& -\frac{B_{2}}{2 n+1} \int_{0}^{R} s^{n+2} h(s) \int_{s}^{R} r^{-n+1} h(r) d r d s \\
& -\frac{B_{3}}{2 n+1} a \int_{0}^{R} h(r)\left(\frac{r}{R}\right)^{n} r^{2} d r+a^{2}\left(B_{4}-\frac{B_{5}}{2 n+1}\right) .
\end{aligned}
$$


Note that $B_{i}>0$. Now we obtain the following result:

Lemma 20. Let

$$
\alpha(x)=h(|x|) Y\left(\frac{x}{|x|}\right),
$$

where $Y(y)$ is a spherical harmonic of degree $n$ with

$$
\int_{S^{2}}|Y(y)|^{2} d y=1
$$

and $r h$ is an integrable function, and let $a \in \mathbb{R}, f(x)=a Y(x /|x|)$. Then

$$
\widehat{V}_{2}\left(\rho_{0}, \alpha, f\right)=V_{2, n}\left(\rho_{0}, h, a\right) .
$$

Proof. Using Lemma 5 we easily obtain

$$
\begin{aligned}
\int_{B_{R}} & \int_{B_{R}} \frac{\alpha(x) \alpha(y)}{|x-y|} d x d y \\
& =\frac{4 \pi}{2 n+1} \int_{0}^{R} \int_{0}^{R} \min \left(\frac{r^{n+2}}{s^{n-1}}, \frac{s^{n+2}}{r^{n-1}}\right) h(s) h(r) d r d s \\
& =\frac{8 \pi}{2 n+1} \int_{0}^{R} \int_{s}^{R} s^{n+2} r^{-n+1} h(r) h(s) d r d s,
\end{aligned}
$$

and also

$$
\begin{aligned}
-\frac{A \rho_{0}^{2}(R) R^{2}}{2 \pi} \int_{\partial B_{R}} \int_{\partial B_{R}} \frac{f(x) f(y)}{|x-y|} d x d y= & -a^{2} \frac{2 R^{5} A \rho_{0}^{2}(R)}{2 n+1} \\
-\frac{A \rho_{0}(R) R}{\pi} \int_{B_{R}} \alpha(y) \int_{\partial B_{R}} \frac{f(x)}{|x-y|} d \sigma_{x} d y= & -\frac{4 A \rho_{0}(R) R^{2}}{2 n+1} \\
& \times a \int_{0}^{R} h(r)\left(\frac{r}{R}\right)^{n} r^{2} d r .
\end{aligned}
$$

Then the proof is immediate.

Now it is easy to see using again Lemma 5 that for the second variation the following is true. If $\left\{Y_{i}\right\}_{i=1}^{\infty}$ is a complete orthonormal basis of $L_{2}\left(S^{2}\right)$ of spherical harmonics of degree $n(i)$ and

$$
\alpha(x)=\sum_{i=1}^{\infty} h_{i}(|x|) Y_{i}\left(\frac{x}{|x|}\right), \quad f(x)=\sum_{i=1}^{\infty} a_{i} Y_{i}(x),
$$


then we have

$$
\widehat{V}_{2}\left(\rho_{0}, \alpha, f\right)=\sum_{i=1}^{\infty} V_{2, n(i)}\left(\rho_{0}, h_{i}, a_{i}\right) .
$$

Now we can consider each of these expressions separately. So therefore now let $f(x)=a Y(x)$ and $\alpha(x)=h(|x|) Y(x /|x|)$ where $Y$ has $L_{2}$-norm one on $S^{2}$ and is of degree $n$. The following lemma summarizes the result of the previous chapter needed here and draws one important conclusion in the case $n=0$. Refer back to equation (7) in Definition 2, which then gives the condition

$$
-a R \rho_{0}(R)=\int_{0}^{R} h(r) r^{2} d r
$$

for $n=0$. Note also that for $n>0$ the integral condition (7) is automatically fulfilled.

Lemma 21. Given $M>0$ and $p_{0}>0$ there is exactly one $R_{0}>0$ where $e(R)$ takes its minimum and one function $\rho_{0} \in C^{\infty}\left(\left[0, R_{0}\right]\right)$ such that $e\left(R_{0}\right)=E\left(B_{R_{0}}, \rho_{0}\right) \leq E\left(B_{R^{\prime}}, \rho\right)$ for all $R^{\prime}, \rho \in A\left(R^{\prime}, M\right)$. The function $s(r)=\left(\rho_{0}(r)\right)^{\varkappa-1}$ belongs to $C^{2}\left(\left[0, R_{0}\right]\right)$, fulfills the equation (4) there, and $\rho_{0}^{\varkappa}\left(R_{0}\right)=p_{0}$. For $h$ with $h(|x|) \in L_{2}\left(B_{R_{0}}\right)$ and $a \in \mathbb{R}$ fulfilling (15), we also have

$$
V_{2,0}\left(\rho_{0}, h, a\right) \geq \beta\left(a^{2}+\int_{0}^{R_{0}} h^{2}(r) r^{2} d r\right)
$$

with $a \beta>0$.

Proof. Most of this lemma was already proved in the previous section. We only need to prove the strict positivity of the variation. We will now split our considerations up into two parts. First we will deal with the variations that leave the domain the same, i.e. $a=0$. From (15) and $a=0$ we get $\int_{0}^{R} h(r) r^{2} d r=0$. We extend $\rho_{0}$ by means of the Lane-Emden equation up to the radius where it becomes zero. Defining

$W(h, R)=\varkappa(\varkappa-1) \int_{0}^{R} \rho_{0}^{\varkappa-2} h^{2} r^{2} d r-2 A \int_{0}^{R} \int_{0}^{R} \min \left(r s^{2}, r^{2} s\right) h(s) h(r) d r d s$ we get $V_{2,0}\left(\rho_{0}, h, 0\right)=W\left(h, R_{0}\right)$, also using (14). We want to show there is a $\delta>0$ such that

$$
V_{2,0}\left(\rho_{0}, h, 0\right) \geq \delta \int_{0}^{R} \rho_{0}^{\varkappa-2} h^{2} r^{2} d r \text { for } h \text { with } \int_{0}^{R} h(r) r^{2} d r=0 .
$$

This is equivalent to showing that

$$
\inf _{h \in A F\left(R_{0}\right)} W\left(h, R_{0}\right)>0
$$


with

$$
A F(R)=\left\{h: \int_{0}^{R} \rho_{0}^{\varkappa-2} h^{2}(r) r^{2} d r=1 \text { and } \int_{0}^{R} h(r) r^{2} d r=0\right\} .
$$

As the solution of the Lane-Emden equation minimizes the energy for its mass for all radii, we get that

$$
\inf _{h \in A F\left(R_{0}\right)} W\left(h, R_{0}\right) \geq 0
$$

for all $R_{0}<R_{1}$. Returning the integrals to three-dimensional space we can easily see by means of the arguments used in Lemma 18 that there is a function $h_{0} \in$ $A F\left(R_{0}\right)$ minimizing $W\left(h, R_{0}\right)$ in that set. We just need to show $W\left(h_{0}, R_{0}\right)>0$. If we had $W\left(h_{0}, R_{0}\right)=0$, we could continue $h_{0}$ as zero to any interval $\left[0, R^{\prime}\right]$ with $R_{0}<R^{\prime}<R_{1}$, and we would have $W\left(h_{0}, R^{\prime}\right)=0$ also, so $h_{0}$ would be a minimizer for $R^{\prime}$ also as $W\left(h, R^{\prime}\right) \geq 0$ as long as $\int_{0}^{R^{\prime}} h(r) r^{2} d r=0$. Now all such minimizing functions $h$ fulfill the equations

$$
\begin{gathered}
\varkappa(\varkappa-1) \rho_{0}^{\varkappa-2} h(r)=\frac{2 A}{r^{2}} \int_{0}^{R^{\prime}} \min \left(s^{2} r, r^{2} s\right) h(s) d s+C, \quad \text { and } \\
\varkappa(\varkappa-1)\left(r^{2}\left(\rho_{0}^{\varkappa-2} h(r)\right)^{\prime}\right)^{\prime}=-2 A r^{2} h(r) .
\end{gathered}
$$

Now $h_{0}$ would have to solve this equation and be zero in an entire interval. Thus it would have to be zero everywhere contrary to our assumptions. So we obtain for $h$ with $\int_{0}^{R} h(r) r^{2} d r=0$ that

$$
V_{2,0}\left(\rho_{0}, h, 0\right) \geq \delta \int_{0}^{R} h^{2}(r) r^{2} d r
$$

with a $\delta>0$. It is easy to see that this is still true with $\frac{\delta}{2}$ instead of $\delta$ for $h$ with

$$
\left|\int_{0}^{R} h(r) r^{2} d r\right| \leq \eta\left(\int_{0}^{R} h^{2}(r) r^{2} d r\right)^{1 / 2}
$$

for a, potentially very small, $\eta>0$. For all variations fulfilling (15), this also means

$$
|a| \leq \eta C\left(\int_{0}^{R} h^{2}(r) r^{2} d r\right)^{1 / 2}
$$

Thus even 


$$
\begin{gathered}
V_{2,0}\left(\rho_{0}, h, a\right)=B_{1} \int_{B_{R}} \rho_{0}^{\varkappa-2} h^{2} r^{2} d r \\
-\frac{B_{2}}{2} \int_{0}^{R} \int_{0}^{R} \min \left(r s^{2}, r^{2} s\right) h(s) h(r) d r d s \\
-B_{3} a \int_{0}^{R} h(r) r^{2} d r+a^{2}\left(B_{4}-B_{5}\right) \\
\geq \frac{\delta}{4} \int_{0}^{R} h^{2}(r) r^{2} d r
\end{gathered}
$$

if $\eta>0$ is just chosen small enough. Now we consider the case

$$
|a| \geq \eta C\left(\int_{0}^{R} h^{2}(r) r^{2} d r\right)^{1 / 2}
$$

We have of course that

$$
I\left(\rho_{0}+t \varphi, \mathrm{id}+\sqrt{4 \pi} t a Y\right) \geq e\left(R_{0}+a t\right),
$$

and as both functions have the derivative zero at $t=0$, we get

$$
4 \pi V_{2,0}\left(\rho_{0}, h, a\right) \geq e^{\prime \prime}\left(R_{0}\right) a^{2} \geq C\left(a^{2}+\int_{0}^{R} h^{2}(r) r^{2} d r\right)
$$

with a $C>0$.

To continue our argument we need the following result:

Lemma 22. For $n \geq 1$ we have

$$
V_{2, n}\left(\rho_{0}, h, a\right) \geq \frac{3}{2 n+1} V_{2,1}\left(\rho_{0},|h|,|a|\right)+\frac{2(n-1)}{2 n+1}\left(B_{1} \int_{B_{R}} \rho_{0}^{\varkappa-2} h^{2} r^{2} d r+a^{2} B_{4}\right) .
$$

Proof. We have

$$
\int_{0}^{R} s^{n+2} h(s) \int_{s}^{R} r^{-n+1} h(r) d r d s \leq \int_{0}^{R} s^{n+2}|h(s)| \int_{s}^{R} r^{-n+1}|h(r)| d r d s
$$

and so 


$$
\begin{aligned}
V_{2, n}\left(\rho_{0}, h, a\right) \geq B_{1} \int_{B_{R}} \rho_{0}^{\varkappa-2} h^{2} r^{2} d r \\
\quad-\frac{B_{2}}{2 n+1} \int_{0}^{R} s^{n+2}|h(s)| \int_{s}^{R} r^{-n+1}|h(r)| d r d s \\
\quad-\frac{B_{3}|a|}{2 n+1} \int_{0}^{R}|h(r)|\left(\frac{r}{R}\right)^{n} r^{2} d r+a^{2}\left(B_{4}-\frac{B_{5}}{2 n+1}\right),
\end{aligned}
$$

which implies that

$$
V_{2, n}\left(\rho_{0}, h, a\right) \geq V_{2, n}\left(\rho_{0},|h|,|a|\right) .
$$

Now for $n \geq 1$ we have

$$
\left(\frac{r}{R}\right)^{n} \leq \frac{r}{R}, \text { and } s^{n+2} r^{-n+1}=\left(\frac{s}{r}\right)^{n-1} s^{3} \leq s^{3} \text { for } r \geq s .
$$

This of course implies

$$
\begin{aligned}
V_{2, n}\left(\rho_{0}, h, a\right) \geq B_{1} \int_{B_{R}} \rho_{0}^{\varkappa-2} h^{2} r^{2} d r-\frac{B_{2}}{2 n+1} \int_{0}^{R} s^{3}|h(s)| \int_{s}^{R}|h(r)| d r d s \\
\quad-\frac{B_{3}|a|}{2 n+1} \int_{0}^{R}|h(r)| \frac{r^{3}}{R} d r+a^{2}\left(B_{4}-\frac{B_{5}}{2 n+1}\right) \\
=\frac{3}{2 n+1} V_{2,1}\left(\rho_{0},|h|,|a|\right)+\frac{2 n-2}{2 n+1}\left(B_{1} \int_{B_{R}} \rho_{0}^{\varkappa-2} h^{2} r^{2} d r+a^{2} B_{4}\right) .
\end{aligned}
$$

Now we want to prove that there is a $\delta>0$ such that

$$
V_{2,1}\left(\rho_{0}, h, 0\right) \geq \delta \int_{B_{R_{0}}} \rho_{0}^{\varkappa-2} h^{2} r^{2} d r \text { and } V_{2,1}\left(\rho_{0}, h, a\right) \geq 0 .
$$

Lemma 23. With

$$
H(s)=\int_{s}^{R} h(r) d r
$$

we have

$$
\begin{aligned}
V_{2,1}\left(\rho_{0}, h, a\right)=B_{1} \int_{B_{R}} \rho_{0}^{\varkappa-2}\left(H^{\prime}(r)\right)^{2} r^{2} d r-\frac{B_{2}}{2} \int_{0}^{R} r^{2} H^{2}(r) d r \\
\quad-B_{3} a R^{-1} \int_{0}^{R} H(r) r^{2} d r+a^{2}\left(B_{4}-\frac{B_{5}}{3}\right) .
\end{aligned}
$$


Proof. As $H^{\prime}(s)=-h(s)$ and $H(R)=0$ we get

$$
\begin{aligned}
\int_{0}^{R} s^{3} h(s) \int_{s}^{R} h(r) d r d s & =-\int_{0}^{R} s^{3} H^{\prime}(s) H(s) d s \\
& =-\int_{0}^{R} s^{3} \frac{1}{2} \frac{d}{d s} H(s)^{2} d s=\frac{3}{2} \int_{0}^{R} s^{2} H(s)^{2} d s .
\end{aligned}
$$

In addition,

$$
\int_{0}^{R} h(r) \frac{r^{3}}{R} d r=-\int_{0}^{R} H^{\prime}(r) \frac{r^{3}}{R} d r=3 R^{-1} \int_{0}^{R} H(r) r^{2} d r .
$$

Finally

$$
\int_{B_{R}} \rho_{0}^{\varkappa-2} h^{2} r^{2} d r=\int_{B_{R}} \rho_{0}^{\varkappa-2}\left(H^{\prime}(r)\right)^{2} r^{2} d r
$$

Putting it all together we get our claim.

Now we can prove the following lemma.

Lemma 24. For $a \in \mathbb{R}$ and $h \in C^{1}\left(\left[0, R_{0}\right]\right)$ we have

$$
V_{2,1}\left(\rho_{0}, h, 0\right) \geq \delta \int_{0}^{R_{0}} h^{2}(r) r^{2} d r \text { and } V_{2,1}\left(\rho_{0}, h, a\right) \geq 0
$$

with some $\delta>0$.

Proof. We start by proving the first inequality. We have

$$
V_{2,1}\left(\rho_{0}, h, 0\right)=B_{1} \int_{B_{R_{0}}} \rho_{0}^{\varkappa-2}\left(H^{\prime}(r)\right)^{2} r^{2} d r-\frac{B_{2}}{2} \int_{0}^{R_{0}} r^{2} H^{2}(r) d r
$$

as we just calculated. Note $B_{1}=\varkappa(\varkappa-1)$ and $B_{2}=4 A$. To check that this always positive unless $H \equiv 0$ we consider

$$
\varkappa(\varkappa-1) \int_{0}^{R_{0}} r^{2} \rho_{0}^{\varkappa-2}(r)\left(H^{\prime}(r)\right)^{2} d r\left[2 A \int_{0}^{R_{0}} s^{2} H(s)^{2} d s\right]^{-1} .
$$

If we can show the infimum of this quotient is larger than 1 for all $H$ with $H(R)=0$, then we are done. It is easily seen that it equals

$$
\frac{\varkappa(\varkappa-1) \int_{B_{R}} \rho_{0}^{\varkappa-2}(|x|)|\nabla g|^{2} d x}{2 A \int_{B_{R}} g^{2} d x}
$$


with $g(x)=H(|x|)$. This is the Rayleigh quotient for the eigenvalue problem associated with the Dirichlet problem for the operator

$$
L g=\frac{\varkappa(\varkappa-1)}{2 A} \operatorname{div}\left(\rho_{0}^{\varkappa-2}(|x|) \nabla g\right)
$$

on $B_{R}$. (Remember $H(R)=0$.) The minimum, which could not be any bigger than that for radially symmetric functions, is therefore obtained by a function $f$ regular at the origin which is strictly positive in the interior, and equals zero on the boundary. Now due to equation 1 the function $g=\rho_{0}(|x|)$ is easily seen to solve the equation $L g=-g$ and be strictly positive in the set $\bar{B}_{R}$. If the minimum of the quotient were indeed smaller than or equal to 1 , then the minimizer $f$ would fulfill the equation $L f=-\lambda f$ with a $\lambda \leq 1$. The following easy comparison argument shows this cannot be true. For $\mu>0$ we have

$$
L(f-\mu g)=-\lambda f+\mu g=-(f-\mu g)+(1-\lambda) f \geq-(f-\mu g) .
$$

So the function $f-\mu g$ cannot take a nonpositive maximum in $B_{R}$. If $\mu$ is sufficiently large, we have $f-\mu g \leq 0$ in all of $B_{R}$. If this is true then

$$
f(y)-\mu g(y) \leq \max _{x \in \partial B_{R}}(f(x)-\mu g(x))=-\mu \min _{x \in \partial B_{R}}(g(x)) \text { for all } y \in B_{R} .
$$

Thus

$$
f(y)-\mu g(y) \leq-\mu \min _{x \in \partial B_{R}}(g(x))<0,
$$

and so the set of all $\mu>0$ for which $f-\mu g \leq 0$ is open. It is of course also relatively closed in $(0, \infty)$, and so

$$
f(y) \leq-\mu \min _{x \in \partial B_{R}}(g(x))+\mu g(y)
$$

for all $\mu>0$. Thus $f \leq 0$ contrary to our assumptions. This proves the first inequality.

As we are considering $V_{2,1}$, we must, by Definitions 2 and 4 , deal with $u(x)=$ $a R^{-4} x|x|^{4} u_{0} \cdot x /|x|$ and $\alpha(x)=h(|x|) u_{0} \cdot x /|x|$, where $u_{0}$ is a constant vector with $\left|u_{0}\right|^{2}=3$, as $u_{0} \cdot x$ is the most general spherical harmonic of norm one and order one on the unit sphere. It is easy to see that then $\varphi=\alpha+\operatorname{div}\left(\rho_{0} u\right)$ also is of the form $b(|x|) u_{0} \cdot x$ if $\alpha$ is. In Theorem 2 we found that the variation actually only depends on $\alpha$ and on $u \cdot n=a u_{0} \cdot x=R_{0} a u_{0} \cdot n$ on $\partial B_{R_{0}}$. So for fixed $\alpha$ this $u$ produces the same value as $R_{0} a u_{0}$. So we now consider $V_{2}\left(\rho_{0}, a u_{0}, \varphi\right)$. Then $J_{T_{t}}=1$ and $T_{t}(x)-T_{t}(y)=x-y$, and so one immediately sees that

$$
I\left(\rho(., t) \cdot T_{t}\right)=I(\rho(., t), \mathrm{id}),
$$

the energy is therefore for all $t$ the same as if the variation of the domain had not even taken place. This is of course the translation-invariance of the energy. This immediately implies

$$
V_{2}\left(\rho_{0}, R_{0} a u_{0}, \varphi\right)=V_{2}\left(\rho_{0}, 0, \varphi\right) \geq 0
$$


using what we just proved. As $V_{2}$ only depends on $u \cdot n$ on $\partial B_{R_{0}}$ and on $\varphi-$ $\operatorname{div}\left(\rho_{0} u\right)$, we get with $\alpha(x)=\varphi(x)-\operatorname{div}\left(\rho_{0} u\right)(x)=h(|x|) u_{0} \cdot x /|x|$,

$$
V_{2,1}\left(\rho_{0}, h, a\right)=\widehat{V}_{2}\left(\rho_{0}, \varphi-\operatorname{div}\left(R_{0} a \rho_{0} u_{0}\right), a u_{0} \cdot x\right)=V_{2}\left(\rho_{0}, R_{0} a u_{0}, \varphi\right) \geq 0,
$$

which is the remainder of our claim.

So, as a final result of our efforts so far, we obtain the following:

Theorem 5. If $\rho_{0}$ is the unique spherically symmetric stationary solution of our problem, then

$$
\widehat{V}_{2}\left(\rho_{0}, \alpha, f\right) \geq \delta\left[\int_{B_{R_{0}}} \alpha^{2} d x+\int_{\partial B_{R_{0}}}|f|^{2} d x\right]
$$

if $f$ is perpendicular to all spherical harmonics of order one.

Proof. Assume

$$
\begin{array}{r}
f=\sum_{i=1}^{\infty} a_{i} Y_{i}\left(\frac{x}{R}\right), \quad \text { and } \\
\alpha(x)=\sum_{i=1}^{\infty} h_{i}(|x|) Y_{i}\left(\frac{x}{|x|}\right) .
\end{array}
$$

By assumption we have $a_{i}=0$ if $n(i)=1$. By Lemmas 22 and 24 we get

$$
\begin{aligned}
& V_{2, n}\left(\rho_{0}, h_{i}, a_{i}\right) \\
& \quad \geq \frac{3}{2 n+1} V_{2,1}\left(\rho_{0},\left|h_{i}\right|,\left|a_{i}\right|\right)+\frac{2(n-1)}{2 n+1}\left(B_{1} \int_{B_{R}} \rho_{0}^{\varkappa-2} h_{i}^{2} r^{2} d r+a_{i}^{2} B_{4}\right) \\
& \quad \geq \vartheta\left(B_{1} \int_{B_{R}} \rho_{0}^{\varkappa-2} h_{i}^{2} r^{2} d r+a_{i}^{2} B_{4}\right)
\end{aligned}
$$

with a $\vartheta>0$. For $n>1$ we just used the fact $V_{2,1}\left(\rho_{0},\left|h_{i}\right|,\left|a_{i}\right|\right) \geq 0$, and for $n=1$ we of course know $a_{i}=0$ for all $i$ with $n(i)=1$, and then we get the inequality from the previous Lemma also. Adding all these expressions up, we get our claim. lemma.

Before we can approach our final problem, we have to prove the following 
Lemma 25. Assume $\Omega$ is close to $B_{R_{0}}$. Then there is a $v_{0}$ such that if $\Omega+v_{0}$ is represented as $\Omega+v_{0}=T\left(B_{R_{0}}\right)$ with a $T$ of the form

$$
T(x)=\left(1+\left(\frac{|x|}{R_{0}}\right)^{4} f\left(\frac{x}{|x|}\right)\right) x,
$$

then $f$ is perpendicular to all spherical harmonics of order one.

Proof. Consider the function

$$
F(y)=\int_{\Omega} \frac{1}{|x-y|} d x .
$$

It of course belongs to $C^{1}\left(\mathbb{R}^{3}\right)$ and is twice differentiable outside $\partial \Omega$. If $\Omega=B_{R_{0}}$ then it has its maximum at zero, and its Hessian matrix is strictly negative there. If $\Omega$ is deformed only a little, the maximum therefore will still be close to zero at a point $y_{0}$, and there

$$
0=\nabla F\left(y_{0}\right)=\int_{\Omega} \frac{x-y_{0}}{\left|x-y_{0}\right|^{3}} d x .
$$

Take $v_{0}=-y_{0}$ and represent $\Omega+v_{0}$ in the form $\Omega+v_{0}=T\left(B_{R_{0}}\right)$ with $T$ as in the statement of the lemma, which is always possible if $\Omega$ is sufficiently close to $B_{R_{0}}$, to obtain

$$
\begin{aligned}
0 & =\int_{\Omega} \frac{x_{i}+v_{0 i}}{\left|x+v_{0}\right|^{3}} d x \\
& =\int_{\Omega+v_{0}} \frac{x_{i}}{|x|^{3}} d x \\
& =\int_{\xi \in S^{2}} \int_{0}^{R_{0}(1+f(\xi))} \frac{r \xi_{i}}{r^{3}} r^{2} d r d \sigma_{\xi} \\
& =\int_{\xi \in S^{2}} \xi_{i} R_{0}(1+f(\xi)) d \sigma_{\xi} \\
& =R_{0} \int_{\xi \in S^{2}} \xi_{i} f(\xi) d \sigma_{\xi} .
\end{aligned}
$$

As the functions $\xi_{i}$ form a basis for the spherical harmonics of order one, we have proved our claim.

So now we confine ourselves to such $T$ and $u$ as we just prove exist in the previous lemma. 
From Theorem 5 we obtain

$$
V_{2}\left(\rho_{0}, \varphi, u\right) \geq \delta_{1}\left[\int_{B_{R_{0}}} \varphi^{2} d x+\int_{B_{R_{0}}}|u|^{2} d x\right]
$$

with a $\delta_{1}>0$. Now from Taylor's theorem we have

$$
\begin{aligned}
& I\left(\rho_{0}+\varphi, \mathrm{id}+u\right) \\
= & I\left(\rho_{0}, \mathrm{id}\right)+\frac{1}{2} V_{2}\left(\rho_{0}, \mathrm{id}, \varphi, u\right)+\int_{0}^{1}\left[\frac{d^{3}}{(d t)^{3}} I\left(\rho_{0}+t \varphi, \mathrm{id}+t u\right)\right]{\frac{(1-t)^{2}}{2} d t}_{\geq} I\left(\rho_{0}, \mathrm{id}\right)+\frac{1}{2} V_{2}\left(\rho_{0}, \mathrm{id}, \varphi, u\right)-C\left(\|\varphi\|_{C^{0}}+\|u\|_{H_{2}^{2}}+\|u\|_{C^{1}}\right)\left(\|u\|_{L_{2}}^{2}+\|\varphi\|_{L_{2}}^{2}\right) \\
\geq & I\left(\rho_{0}, \mathrm{id}\right)+\frac{\delta_{1}}{2}\left[\int_{B_{R}} \varphi^{2} d x+\int_{B_{R}} u^{2} d x\right]-\frac{\delta_{1}}{4}\left[\int_{B_{R}} \varphi^{2} d x+\int_{B_{R}}|u|^{2} d x\right] \\
= & I\left(\rho_{0}, \mathrm{id}\right)+\frac{\delta_{1}}{4}\left(\int_{S^{2}} f^{2} d \sigma+\int_{B_{R}} \varphi^{2} d x\right)
\end{aligned}
$$

for $\|\varphi\|_{C^{0}}+\|u\|_{H_{2}^{2}}+\|u\|_{C^{1}}$ sufficiently small. This gives our claim.

\section{REFERENCES}

[1] A. A. Antonov. Remarks on the problem of stability in stellar dynamics, Soviet Astronomy, 4 (1960), 859-867.

[2] J. Batt, W. Faltenbacher, And E. Horst. Stationary spherically symmetric models in stellar dynamics, Arch. Rat. Mech. Anal. 93 (1986), 159-183.

[3] J. Batt, K. Pfaffelmoser. On the radius continuity of the models of polytropic gas spheres which correspond to the positive solutions of the generalized EmdenFowler equation, Math. Meth. in the Appl. Sci. 10 (1988). 499-516.

[4] F. BAvaud. Equilibrium properties of the Vlasov functional: The generalized Poisson-Boltzmann-Emden equation, Reviews of Modern Physics, 63 (1991), 129149.

[5] W. J. van den Broek and F. Verhulst. A generalized Lane-Emden-Fowler equation, Math. Meth. in the Appl. Sci. 4 (1982) 259-271.

[6] S. Chandrasekhar. An introduction to the study of stellar structure, Univ. of Chicago Press, 1939.

[7] R. Emden. Gaskugeln, Teubner, Leipzig, 1907.

[8] A. Friedman. Partial Differential Equations, Krieger, Malabar, FL, 1976.

[9] B. Gidas, Wei Ming Ni, And L. Nirenberg. Symmetry of positive solutions of nonlinear elliptic equations in $\mathbb{R}^{n}$, Adv. in Math. Suppl. Stud. 7a. Academic Press, New York-London, 1981.

[10] D. Gilbarg And N. S. Trudinger. Elliptic differential equations of second order, Springer, Berlin, 1983. 
[11] H. G. KAPER AND M. K. Kwong. A non-oscillation theorem for the EmdenFowler equation-ground states for semilinear elliptic equations with critical exponents, Journal of Differential Equations 75 (1988) 158-185.

[12] J. H. LANE. On the theoretical temperature of the sun; under the hypothesis of a gaseous mass maintaining its volume by its internal heat, and the laws of gases as known to terrestrial experiment. The American Journal of Science and Arts, Second Series 4, (1870), 57-74.

[13] S. LAng. Differential Manifolds, Springer, New York, 1985.

[14] D. Lynden-Bell And N. SAnitT. The Schrödinger operator criterion for the stability of galaxies and gas spheres, Mon. Not. R. astr. Soc., 143 (1969), 167187.

[15] G. P. HoREdT. Exact solutions of the Lane-Emden equation in $N$-dimensional space, Astronomy and Astrophysics 160 (1986), 148-156.

[16] S. G. Michlin. Partielle Differentialgleichungen der Mathematischen Physik, Harri Deutsch, Thun, Frankfurt, 1978.

[17] S. G. Mikhlin. Mathematical Physics: An Advanced Course, North Holland, Amsterdam, 1970.

[18] E. S. Noussair AND C. A. Swanson. Positive $L_{q}\left(\mathbb{R}^{n}\right)$ solutions of subcritical Emden-Fowler problems, Arch. Rat. Mech. Anal. 101 (1988) 85-93.

[19] M. V. Penston. Dynamics of self-gravitating gas spheres II, Mon. Not. R. Astr. Soc., 145 (1969), 457-485.

[20] G. Sansone. Sulle soluzione di Emden dell'equazione di Fowler, Rend. Mat. Roma 1 (1940) 163-176.

[21] P. SecChi And A. VAlLi. A free boundary problem for compressible viscous fluids, J. Reine u. Angew. Math. 341, 1-31 (1983).

[22] P. SECCHI. On the evolution equation of viscous gaseous stars, Ann. Scuola Norm. Sup. Pisa Cl. Sci. (4), 18 (1991), 295-318.

[23] V. I. Smirnov. A Course of Higher Mathematics, Pergamon, Oxford, New York, 1964.

[24] V. A. Solonnikov And A. TANi. Free boundary problem for a viscous compressible flow with a surface tension, in: 'Constantine Carathéodory: An International Tribute', Vol I, II, pp. 1270-1303, World Scientific Publishing, Teaneck, N.J., 1991.

[25] G. Ströhmer AND W. ZaJACZKOWski. On stability of certain equilibrium solutions for compressible barotropic viscous self-gravitating fluid motions bounded by a free surface, to appear in 'Nonlinear Analysis'.

[26] G. Ströhmer AND W. ZAJACZKOWSKi. Local existence of solutions of free boundary problem for the equations of compressible barotropic viscous self-gravitating fluids, Preprint.

[27] H. TrIEBEL. Interpolation Theory, Function Spaces, Differential Operators, North Holland, Amsterdam, 1978.

[28] G. Wolansky. On the evolution of self-interacting clusters and applications to semilinear equations with exponential nonlinearity, Journal d'Analyse Mathématique, 59 (1992), 251-272.

[29] G. Wolansky. On steady distributions of self-attracting clusters under friction and fluctuations, Arch. Rational Mech. Anal. 119 (1992), 355-391.

[30] J. S. W. Wong. On the generalized Emden-Fowler equation, SIAM Review 17 (1975), 339-360. 
[31] W. M. ZaJaczkowski. On local motion of a compressible barotropic viscous fluid bounded by a free surface, Banach Center Publ. 27 (1992), 511-553, Warsaw, Poland.

[32] W. M. ZAJACZKOWSKI. On nonstationary motion of a compressible barotropic viscous fluid bounded by a free surface, Dissert. Math. 324 (1993), 1-101.

[33] W. M. ZAJACZKowski. On nonstationary motion of a compressible barotropic viscous capillary fluid bounded by a free surface, SIAM J. of Math. Anal. 25 (1994) 1-84.

[34] W. M. ZaJaczKowski. Existence of local solutions for viscous compressible barotropic fluids, Ann. Polon. Math. 60 (1995), 255-287.

The work of the second author was supported, in part, by Polish Grant KBN 2 P301 00806 .

Gerhard Ströhmer

Department of Mathematics

University of Iowa

Iowa City, IA, 52242

U. S. A

E-MAIL: strohmer@math.uiowa.edu
WOJCIECH ZAJACZKOWSKI

Mathematical Institute

Polish Academy of the Sciences

ul. Sniadeckich 8

00-950 Warsaw, POLAND

Received: December 19th, 1996; revised: March 3rd, 1998. 